\title{
Soft matters, or the recursions with massive spinors
}

\author{
Adam Falkowski $^{a}$ and Camila S. Machado ${ }^{b}$ \\ ${ }^{a}$ Université Paris-Saclay, CNRS/IN2P3, IJCLab, \\ 91405 Orsay, France \\ ${ }^{b}$ PRISMA ${ }^{+}$Cluster of Excellence Mainz Institute for Theoretical Physics, \\ Johannes Gutenberg-Universität Mainz, \\ 55099 Mainz, Germany \\ E-mail: adam.falkowski@cern.ch, camila.samachado@gmail.com
}

ABSTRACT: We discuss recursion relations for scattering amplitudes with massive particles of any spin. They are derived via a two-parameter shift of momenta, combining a BCFWtype spinor shift with the soft limit of a massless particle involved in the process. The technical innovation is that spinors corresponding to massive momenta are also shifted. Our recursions lead to a reformulation of the soft theorems. The well-known Weinberg's soft factors are recovered and, in addition, the subleading factors appear reshaped such that they are directly applicable to massive amplitudes in the modern on-shell language. Moreover, we obtain new results in the context of non-minimal interactions of massive matter with photons and gravitons. These soft theorems are employed for practical calculations of Compton and higher-point scattering. As a by-product, we introduce a convenient representation of the Compton scattering amplitude for any mass and spin.

KeYwords: Scattering Amplitudes, Effective Field Theories

ArXiv EPrint: 2005.08981 


\section{Contents}

1 Introduction 1

2 Spinor conventions $\quad 4$

3 Soft recursions 5

3.1 Momentum shift 6

3.2 Recursion 6

$\begin{array}{lll}3.3 & \text { Spinor shift } & 7\end{array}$

4 Soft theorems $\quad 9$

4.1 Minimal gravity 9

$\begin{array}{lll}4.2 & \text { Non-minimal gravity } & 12\end{array}$

$\begin{array}{lll}4.3 & \text { Photons } & 13\end{array}$

5 Applications $\quad \mathbf{1 5}$

$\begin{array}{lll}5.1 & \text { Compton scattering } & 15\end{array}$

$\begin{array}{ll}5.2 \text { Gravitational Compton scattering } & 18\end{array}$

$\begin{array}{lll}5.3 & \text { Higher-point } & 19\end{array}$

6 Exponential representation $\quad 20$

6.1 Photon exponentiation 21

6.2 Soft exponentiation 22

$\begin{array}{lll}7 & \text { Conclusions } & 23\end{array}$

$\begin{array}{ll}\text { A Summary of spinor shifts } & 24\end{array}$

B UV terms in Compton scattering $\quad 26$

B.1 Electromagnetic Compton scattering 26

$\begin{array}{ll}\text { B.2 Gravitational Compton scattering } 28 & 28\end{array}$

\section{Introduction}

Soft theorems describe general properties of scattering amplitudes in the infrared (IR) regime where the (four-)momentum of one massless particle approaches zero, $p^{\mu} \rightarrow \epsilon p^{\mu}$ with $\epsilon \rightarrow 0$. In this limit it is possible to write down a recursion relation connecting 
the amplitude to a lower-point one with the soft particle removed. For example, for soft photons with helicity $h= \pm 1$ and soft gravitons with $h= \pm 2$ the recursions take the form

$$
\mathcal{M}_{n+1}^{ \pm 1}=\left(\frac{S_{ \pm 1}^{(0)}}{\epsilon^{2}}+\frac{S_{ \pm 1}^{(1)}}{\epsilon}\right) \mathcal{M}_{n}, \quad \mathcal{M}_{n+1}^{ \pm 2}=\left(\frac{S_{ \pm 2}^{(0)}}{\epsilon^{3}}+\frac{S_{ \pm 2}^{(1)}}{\epsilon^{2}}+\frac{S_{ \pm 2}^{(2)}}{\epsilon}\right) \mathcal{M}_{n}
$$

$S_{ \pm h}^{(i)}$ are called the soft factors. They are universal, in the sense that they do not depend on what other "hard" particles are involved in the scattering.

Since the seminal works of Low [1] and Weinberg [2], which explored the leading term in the soft momentum expansion, the importance of the soft theorems has been revealed through applications that span very different fields. In particular, soft theorems turn out to be intricately related to the consistency conditions of the S-matrix such as gauge invariance, locality and unitarity [3, 4]. Furthermore, it was shown that they can be understood as Ward identities of asymptotic symmetries [5]. They have also been relevant to understanding the landscape of effective field theories (EFTs) (see e.g. [6-10]).

A new life into the study of soft theorems was brought by the emergence of on-shell methods for calculating scattering processes. These are founded on the spinor techniques, which make manifest the little group transformation properties of the amplitudes. An important step in this development was ref. [11] by Cachazo and Strominger. Using the BCFW recursion relations [12] they were able to extend the graviton soft theorems up to sub-subleading order at tree level. ${ }^{1}$ Subsequently, the work by Elvang et al. [16] extended on-shell soft theorems to a broad class of theories with massless particles. This is achieved thanks to a modified recursion relation that combines double BCFW and soft shifts. This approach leads to an elegant proof that various S-matrix consistency conditions, such as charge conservation and the equivalence principle, follow directly from Poincaré invariance, locality, and unitarity, without ever mentioning gauge invariance. Moreover, ref. [16] systematized the classes of effective operators that modify the soft theorems at the subleading order for photons and the sub-subleading order for gravitons.

Despite these rapid developments, all of the results derived with on-shell techniques are valid only for massless theories. ${ }^{2}$ Meanwhile, a convenient spinor formalism for dealing with massive particles was introduced in [22]. There are several motivations to rewrite the soft theorems such that they can be directly applied to theories using the on-shell language and massive spinors. Perhaps the most pressing is the growing importance of the on-shell program in the context of black hole computations [23-26]. Another is the progress in on-shell formulation of massive EFTs, such as e.g. massive gravity [27-30], or the Standard Model EFT [31-33]. More generally, evolution of on-shell tools to deal with massive theories may reveal unexpected simplicity of their amplitudes, much as it happened for massless theories.

In this paper, we establish soft recursion relations that are valid for general theories with massive particles of any spin, extending the results of [16]. This is achieved by introducing a new kind of holomorphic spinor shift, where also massive spinors are shifted in

\footnotetext{
${ }^{1}$ In this paper we restrict the discussion to tree level. Although the leading (Weinberg's) soft factor is not modified at loop level, the story for the sub-leading orders is more subtle [13-15].

${ }^{2}$ For soft theorems with massive particles using the effective action formalism, see e.g. [17-21].
} 
addition to massless ones. This allows us to derive a general recursion relation that remains valid even when the soft particle is the only massless particle in the scattering process. Our formula can be applied to describe emission of a soft particle of any helicity $|h| \leq 2$. In this paper we work out the resulting soft theorems for photons and gravitons. These have the same general structure as in the standard derivation, however they can be directly applied to amplitudes written in term of massive and massless spinors. In particular, the subleading soft factors become differential operators acting on the massive spinors and on their little group indices. We explicitly connect the subleading soft theorems to multipole expansion of the 3-point interaction between massive matter and photons/gravitons. As a corollary, we obtain a compact and elegant proof that gravitational dipole interactions of fundamental particles of any spin are inconsistent with the assumption of Poincaré invariance, locality, and unitarity.

We also discuss a couple of concrete applications of the soft recursion relations in massive theories. One is for the simple 4-body process of Compton scattering of photons and gravitons. For any spin of the matter particle, the recursion correctly captures the IR part of the amplitude, that is the one containing the physical poles in the Mandelstam variables. The result agrees with the one in ref. [22] obtained by requiring that the residues of the physical poles factorize into 3-point amplitudes. As discussed in [22, 34], for higher spins the IR part inevitably develops a spurious pole that is not associated with an exchange of a physical particle. Therefore, to make it consistent with unitarity, the Compton amplitude has to be augmented by a UV term that cancels the spurious pole without affecting the physical ones. In our derivation, this UV term arises as a boundary term in the recursion relation. As an aside, we present a systematic algorithm to build the UV term in Compton scattering, alternative to the one in [34]. We also make some baby steps toward higher-point amplitudes of any spin, by calculating specific 5-point amplitudes with 3 photons or 3 gravitons.

Finally, we touch on the subject of exponential representation of soft amplitudes. Exponentiated soft factors play an important role in the matching between quantum and classical limits. In particular, they are instrumental in certain calculations of black hole observables [24, 35]. They can also be used to write scattering amplitudes in the Mellin space, which can be connected with CFT correlators in the celestial sphere (see e.g. [3638]). Our recursion relations lead to a simple proof of the exponentiation of Compton amplitudes [24] for any mass and spin. We also remark that the photon and graviton soft factors can be written in terms of an exponential operator in the scattering of massive particles of generic spin, which previously was only done for the minimal-helicity violating (MHV) sector of massless gravity/Yang-Mills [14].

Our paper is organized as follows. The (massless and massive) spinor formalism is briefly reviewed in section 2. The new soft recursions, valid for particles of any mass and spin, are derived in section 3, from which the soft theorems in section 4 directly follow. Some applications for calculating concrete amplitudes are presented in section 5. Section 6 discusses the exponential representation of soft amplitudes and Compton scattering. Section 7 is reserved for conclusions and future directions. Appendix A lists the possible spinor shifts realizing our momentum shift, some of which are not employed in this paper but may be useful for other applications. Appendix B describes in detail our construction of UV/boundary terms in Compton scattering. 


\section{Spinor conventions}

We begin with a brief overview of our conventions (readers well-versed in the massless and massive spinor formalism are invited to skip this section). We work in four dimensions with the mostly-minus metric $\eta_{\mu \nu}=\operatorname{diag}(1,-1,-1,-1)$. The Lorentz algebra can be decomposed into $\mathrm{SL}(2, \mathbb{C}) \times \mathrm{SL}(2, \mathbb{C})$. Holomorphic and antiholomorphic spinors $\psi_{\alpha}$ and $\psi_{\dot{\alpha}}$ transform under the respective $\mathrm{SL}(2, \mathbb{C})$ factors with indices being raised and lowered by the antisymmetric epsilon tensor:

$$
\psi^{\alpha}=\epsilon^{\alpha \beta} \psi_{\beta}, \quad \psi_{\alpha}=\epsilon_{\alpha \beta} \psi^{\beta},
$$

and idem for dotted indices. Vector and spinor Lorentz indices can be traded with the help of the sigma matrices $\left(\sigma^{\mu}\right)_{\alpha \dot{\beta}}=(\mathbb{I}, \vec{\sigma})$ and $\left(\bar{\sigma}^{\mu}\right)^{\dot{\alpha} \beta}=(\mathbb{I},-\vec{\sigma})$, where $\vec{\sigma}$ are the Pauli matrices. Given the momentum ${ }^{3} p_{\mu}$, we can construct the $2 \times 2$ matrices

$$
p_{\mu}\left(\sigma^{\mu}\right)_{\alpha \dot{\alpha}} \equiv(p \sigma)_{\alpha \dot{\alpha}}, \quad p_{\mu}\left(\bar{\sigma}^{\mu}\right)^{\dot{\alpha} \alpha} \equiv(p \bar{\sigma})^{\dot{\alpha} \alpha} .
$$

These naturally act on the spinor indices: $(p \bar{\sigma}) \psi \equiv(p \bar{\sigma})^{\dot{\alpha} \beta} \psi_{\beta},(p \sigma) \tilde{\psi} \equiv(p \sigma)_{\alpha \dot{\beta}} \tilde{\psi}^{\dot{\beta}}, \tilde{\psi}(p \bar{\sigma}) \equiv$ $\tilde{\psi}_{\dot{\beta}}(p \bar{\sigma})^{\dot{\beta} \alpha}, \psi(p \sigma) \equiv \psi^{\beta}(p \sigma)_{\beta \dot{\alpha}}$, where repeating spinor indices are implicitly summed over. We will often omit the spinor indices, as with these rules the contractions are always unambiguous.

Massless momentum can be represented by a pair of (commuting) spinors $\lambda_{\alpha}, \tilde{\lambda}_{\dot{\beta}}$ :

$$
(p \sigma)_{\alpha \dot{\beta}}=\lambda_{\alpha} \tilde{\lambda}_{\dot{\beta}}, \quad(p \bar{\sigma})^{\bar{\alpha} \beta}=\tilde{\lambda}^{\dot{\alpha}} \lambda^{\beta},
$$

such that $p^{2}=0$ is automatic for any $\lambda, \tilde{\lambda}$ thanks to the identity $\lambda^{\alpha} \lambda_{\alpha}=0=\tilde{\lambda}_{\dot{\alpha}} \tilde{\lambda}^{\dot{\alpha}}$. It follows that the massless spinors satisfy the Weyl equations: $\lambda(p \sigma)=(p \sigma) \tilde{\lambda}=0$, $(p \bar{\sigma}) \lambda=\tilde{\lambda}(p \bar{\sigma})=0$. For real $p_{\mu}$ we have the additional constraint $\tilde{\lambda}=\bar{\lambda}$, but in general momenta are allowed to be complex, in which case $\tilde{\lambda}$ and $\lambda$ are independent. The little group transformations (i.e. for a fixed $p_{\mu}$, the subset of Lorentz transformations leaving $p_{\mu}$ invariant) correspond to $\mathrm{U}(1)$ acting as $\lambda \rightarrow t^{-1} \lambda, \tilde{\lambda} \rightarrow t \tilde{\lambda}$. The Lorentz-invariant and little group covariant building blocks are commonly represented by the bra-ket notation:

$$
\langle i j\rangle \equiv \lambda_{i}^{\alpha} \lambda_{j \alpha}=\epsilon^{\beta \alpha} \lambda_{i \alpha} \lambda_{j \beta}=\left(\lambda_{i} \lambda_{j}\right), \quad[i j] \equiv \tilde{\lambda}_{i \dot{\alpha}} \tilde{\lambda}_{j}^{\dot{\alpha}}=\epsilon^{\dot{\alpha} \dot{\beta}} \tilde{\lambda}_{i \dot{\alpha}} \lambda_{j \dot{\beta}}=\left(\tilde{\lambda}_{i} \tilde{\lambda}_{j}\right)
$$

and the Lorentz contraction of momenta can be written as $2 p_{i} p_{j}=\langle i j\rangle[j i]^{4}$ The bra-ket notation can be naturally extended to more complicated contractions, e.g. $\left(\lambda_{i} p_{k} \sigma \tilde{\lambda}_{j}\right) \equiv$ $\left\langle i p_{k} j\right],\left(\lambda_{i} p_{k} \sigma p_{l} \bar{\sigma} \tilde{\lambda}_{j}\right) \equiv\left\langle i p_{k} p_{l} j\right]$, etc. In the following, we choose to write uncontracted spinors explicitly as in eq. (2.1), and reserve the bra-ket notation for Lorentz contractions only; e.g. we write $\langle i j\rangle \lambda_{k} \equiv\left(\lambda_{i} \lambda_{j}\right) \lambda_{k}$. The massless spinors can be related to polarization

\footnotetext{
${ }^{3}$ In this paper "momentum" always means four-momentum.

${ }^{4}$ We always follow the conventions of ref. [39]. For the holomorphic contraction $\langle\cdot\rangle$ this differs by a sign from some of the on-shell literature.
} 
tensors in the standard Lagrangian formalism. In particular, the polarization vectors of a massless spin-1 particle can be written in terms of the spinors as

$$
\epsilon_{\mu}^{-}=\frac{\left(\lambda \sigma_{\mu} \tilde{\zeta}\right)}{\sqrt{2}[\lambda \zeta]}, \quad \epsilon_{\mu}^{+}=\frac{\left(\zeta \sigma_{\mu} \tilde{\lambda}\right)}{\sqrt{2}\langle\lambda \zeta\rangle}
$$

where $\tilde{\zeta}$ and $\zeta$ are arbitrary reference spinors representing the gauge freedom. Similarly, for a massless spin-2 particle, the polarization tensors are $\epsilon_{\mu \nu}^{ \pm}=\epsilon_{\mu}^{ \pm} \epsilon_{\nu}^{ \pm}$, where now the freedom of choosing the reference spinors represents general coordinate invariance.

Massive momentum satisfying the on-shell condition $p^{2}=m^{2}$ can be represented by four spinors $\chi_{\alpha}^{1}, \chi_{\alpha}^{2}, \tilde{\chi}_{\dot{\beta} 1}, \tilde{\chi}_{\dot{\beta} 2}$. They can be collected into $\chi^{J}$ and $\tilde{\chi}_{J}$, where $J=1,2$ is identified with the $\mathrm{SU}(2)$ little group index which, in complete analogy to spinor indices, can be raised and lowered by epsilon tensors. The spinors are related to the momentum by the formula

$$
(p \sigma)_{\alpha \dot{\beta}}=\chi_{\alpha}^{J} \tilde{\chi}_{\dot{\beta} J}, \quad(p \bar{\sigma})^{\alpha \dot{\beta}}=\tilde{\chi}_{J}^{\dot{\alpha}} \chi^{\beta J},
$$

where summation over the little group indices is implicit. This is a natural and convenient generalization of the spinor helicity formalism to massive particles [22] (for earlier works, see e.g. [40, 41]). The massive spinors are normalized as

$$
\left(\chi^{J} \chi_{K}\right)=\delta_{K}^{J} m, \quad\left(\tilde{\chi}_{J} \tilde{\chi}^{K}\right)=\delta_{J}^{K} m,
$$

from which follow the Dirac equations

$$
(p \sigma) \tilde{\chi}^{J}=m \chi^{J}, \quad(p \bar{\sigma}) \chi^{J}=m \tilde{\chi}^{J}, \quad \chi^{J}(p \sigma)=-m \tilde{\chi}^{J}, \quad \tilde{\chi}^{J}(p \bar{\sigma})=-m \chi^{J} .
$$

These allow one to trade $\chi$ for $\tilde{\chi}$ and vice-versa. An amplitude describing scattering of $n$ massive incoming particles with spin $S_{i}, i=1 \ldots n$, for each $i$ will contain exactly $2 S_{i}$ spinors $\chi_{i}^{J_{k}}$ or $\tilde{\chi}_{i}^{J_{k}}$ with uncontracted little group indices. Massive outgoing particles can be represented by spinors $\chi_{i J_{k}}$ or $\tilde{\chi}_{i J_{k}}$ with lowered little group indices, however in this paper we treat all external particles as incoming. The little group indices corresponding to the same particle are always implicitly symmetrized over. To reduce clutter, in the following we will often omit the little group indices whenever it does not lead to ambiguities. Lorentz contractions of massive spinors will be shortened via the bra-ket notation:

$$
\langle\mathbf{i j}\rangle \equiv \chi_{i}^{\alpha} \chi_{j \alpha}=\left(\chi_{i} \chi_{j}\right), \quad[\mathbf{i j}] \equiv \tilde{\chi}_{i \dot{\alpha}} \tilde{\chi}_{j}^{\dot{\alpha}}=\left(\tilde{\chi}_{i} \tilde{\chi}_{j}\right),
$$

where we adhere to the bold notation introduced in ref. [22].

\section{Soft recursions}

In this section we derive a formula connecting an $n+1$-point amplitude with at least one massless particle to $n$-point amplitudes. The formula picks up the leading terms in the limit where the momentum of a massless particle is continuously taken to zero. That massless particle is called soft, and the formula is referred to as the soft recursion. The derivation follows similar steps as the one in ref. [16], however it is adapted to allow for massive particles in the scattering process. 


\subsection{Momentum shift}

Consider a set of $n+1$ particles with momenta $p_{0} \ldots p_{n}$ satisfying $\sum_{i=0}^{n} p_{i}=0$ and the on-shell conditions $p_{i}^{2}=m_{i}^{2}$. The particle labeled as 0 is always massless, $p_{0}^{2}=0$, and it will be our soft particle. We perform a two-parameter deformation of this kinematics. One parameter $z$ controls complex deformation of the momenta of the particle 0 and of two other particles, below labeled as $j$ and $k$. The other parameter $\epsilon$ controls the soft limit of the particle 0 . Specifically, the shift is defined as ${ }^{5}$

$\hat{p}_{0}=\epsilon p_{0}-z q_{0}, \quad \hat{p}_{j}=p_{j}-\frac{(\epsilon-1)\left(p_{0} p_{k}\right)-z\left(q_{0} p_{k}\right)}{q_{j} p_{k}} q_{j}, \quad \hat{p}_{k}=p_{k}-\frac{(\epsilon-1)\left(p_{0} p_{j}\right)-z\left(q_{0} p_{j}\right)}{q_{k} p_{j}} q_{k}$,

where $q_{0}, q_{j}, q_{k}$ are four-vectors satisfying

$$
q_{0}^{2}=q_{j}^{2}=q_{k}^{2}=q_{0} p_{0}=q_{j} p_{j}=q_{k} p_{k}=0, \quad q_{j} p_{0}=q_{k} p_{0}=q_{j} q_{0}=q_{k} q_{0}=q_{j} q_{k}=0 .
$$

The first set of equations in eq. (3.2) ensures that the shift does not change the particles' masses, $\hat{p}_{i}^{2}=m_{i}^{2}$, while the role of the second is to ensure shifted momentum conservation, $\sum_{i=0}^{n} \hat{p}_{i}=0$, for any $z$ and $\epsilon$. One advantage of the two-parameter shift in eq. (3.1) compared to simpler BCFW-like one-parameter shifts is that it will directly lead to soft expansion of recursion relations in powers of $1 / \epsilon$. As a bonus, it will allow for more control over the recursions' boundary terms, as the latter will start at higher orders in $1 / \epsilon$.

\subsection{Recursion}

Using the momentum shift in eq. (3.1) one can relate, in a quite model-independent way, $n+1$-point amplitudes in the soft limit to $n$-point amplitudes. Consider an $n+1$ point amplitude $\mathcal{M}_{n+1} \equiv \mathcal{M}(1 \ldots n 0)$. We denote $\hat{\mathcal{M}}_{n+1}^{z, \epsilon}$ its shifted version, which is $\mathcal{M}_{n+1}$ evaluated for the "hatted" kinematics in eq. (3.1). Similarly, we denote $\mathcal{M}_{n} \equiv \mathcal{M}(1 \ldots n)$, and its shifted version by $\hat{\mathcal{M}}_{n}^{z, \epsilon}$. Note that $\hat{\mathcal{M}}^{0,1}=\mathcal{M}$. At tree level $\hat{\mathcal{M}}^{z, \epsilon}$ is a meromorphic function of $z$, with all singularities given by a finite set of simple poles at $z=z_{i}$. Applying the Cauchy formula,

$$
\hat{\mathcal{M}}_{n+1}^{0, \epsilon}=-\sum_{i} \frac{\operatorname{Res}_{z \rightarrow z_{i}} \hat{\mathcal{M}}_{n+1}^{z, \epsilon}}{z_{i}}+B_{\infty}
$$

where the boundary term is $B_{\infty}=\frac{1}{2 \pi i} \int_{C_{\infty}} \frac{\hat{\mathcal{M}}_{n+1}^{z, \epsilon}}{z}$, and $C_{\infty}$ is the circle at infinity. If $\hat{\mathcal{M}}_{n+1}^{z, \epsilon}$ goes to zero as $z \rightarrow \infty$ then $B_{\infty}=0$. The recursion relation is most powerful when $B_{\infty}=0$, but this will not always be true in the cases of interest.

We only focus on a subset of the poles related to the emission of the particle zero from an external leg the $n$-point amplitude $\mathcal{M}_{n}$. In the limit $\epsilon \rightarrow 0$, singular $1 / \epsilon^{n}$ terms in $\hat{\mathcal{M}}_{n+1}^{z, \epsilon}$ can only come from these residues. There is $n$ such poles $z_{l}$ related to the solutions

\footnotetext{
${ }^{5}$ The original reference [16] defines the shift in a slightly different way: $\hat{p}_{j}=p_{j}-\left[\epsilon \alpha_{j}-z \beta_{j}\right] q_{j}, \hat{p}_{k}=$ $p_{k}+\left[\epsilon \alpha_{k}-z \beta_{k}\right] q_{k}$, such that $\hat{p}_{j, k}=p_{j, k}$ for $\epsilon=z=0$. In this version the unshifted kinematics is constrained as $\sum_{i=1}^{n} p_{i}=0$ (as opposed to $\sum_{i=0}^{n} p_{i}=0$ in our case). For this reason we find it more convenient to work with the version in eq. (3.1).
} 
of the equations $\left.\hat{P}_{0 l}^{2}\right|_{z=z_{l}}=m_{l}^{2}$, where $\hat{P}_{0 l} \equiv \hat{p}_{0}+\hat{p}_{l}$ and it is understood that $\hat{p}_{l}=p_{l}$ for $l \neq j, k$. The poles are located at

$$
z_{l}=\epsilon \frac{p_{0} p_{l}}{q_{0} p_{l}}, \quad l=1 \ldots n,
$$

and one can rewrite $\hat{P}_{0 l}^{2}-m_{l}^{2}=-2 q_{0} p_{l}\left(z-z_{l}\right)$. This shows that the poles at $\hat{P}_{0 l}^{2} \rightarrow m_{l}^{2}$ are in one-to-one correspondence with the poles at $z \rightarrow z_{l}$. Unitarity requires that at every pole the amplitude factorizes:

$$
\begin{aligned}
\left.\hat{\mathcal{M}}^{z, \epsilon}(1 \ldots n 0)\right|_{\hat{P}_{0 l}^{2} \rightarrow m_{l}^{2}} & =-\frac{\hat{\mathcal{M}}^{z_{l}, \epsilon}\left(1 \ldots P_{0 l} \ldots n\right) \hat{\mathcal{M}}^{z_{l}, \epsilon}\left(\left(-P_{0 l}\right) l 0\right)}{\hat{P}_{0 l}^{2}-m_{l}^{2}} \\
& =\frac{\hat{\mathcal{M}}^{z_{l}, \epsilon}\left(1 \ldots P_{0 l} \ldots n\right) \hat{\mathcal{M}}^{z_{l}, \epsilon}\left(\left(-P_{0 l}\right) l 0\right)}{2 q_{0} p_{l}\left(z-z_{l}\right)}
\end{aligned}
$$

Thus

$$
\frac{\operatorname{Res}_{z \rightarrow z_{l}} \hat{\mathcal{M}}^{z, \epsilon}(0 \ldots n)}{z_{l}}=\frac{1}{\epsilon} \frac{\hat{\mathcal{M}}^{z_{l}, \epsilon}\left(1 \ldots P_{0 l} \ldots n\right) \hat{\mathcal{M}}^{z_{l}, \epsilon}\left(\left(-P_{0 l}\right) l 0\right)}{2 p_{0} p_{l}} .
$$

Plugging this back into the Cauchy formula in eq. (3.3) on obtains the soft recursion:

$$
\hat{\mathcal{M}}^{0, \epsilon}(1 \ldots n 0)=-\frac{1}{\epsilon} \sum_{l=1}^{n} \frac{\hat{\mathcal{M}}^{z_{l}, \epsilon}\left(1 \ldots P_{0 l} \ldots n\right) \hat{\mathcal{M}}^{z_{l}, \epsilon}\left(\left(-P_{0 l}\right) l 0\right)}{2 p_{0} p_{l}}+\mathcal{O}\left(\epsilon^{0}\right) .
$$

This formula will be the starting point for deriving a new on-shell incarnation of soft theorems which is valid in the presence of massive particles. We will often refer to the $n$-point amplitude above as the hard factor. The boundary term in eq. (3.3) is absorbed in $\mathcal{O}\left(\epsilon^{0}\right)$ above, as it cannot produce singular terms in the $\epsilon \rightarrow 0$ limit.

\subsection{Spinor shift}

We turn to discussing spinor shifts that realize the momentum shift in eq. (3.1). The discussion depends on whether the shifted particles $j$ and $k$ are massive or massless. In the following we first work out the case when $m_{j}>0$ and $m_{k}>0$, and later comment on other possible configurations. Note that $m_{j}$ and $m_{k}$ can be different. Recall also that particle 0 is always massless in this discussion, however other particles with $l \neq 0, j, k$ can be massive or massless, as one pleases.

One possible choice of the shift vectors $q$ in eq. (3.1) is

$$
q_{0} \sigma=y \tilde{\lambda}_{0}, \quad q_{j} \sigma=p_{j} \sigma \tilde{\lambda}_{0} \tilde{\lambda}_{0}, \quad q_{k} \sigma=p_{k} \sigma \tilde{\lambda}_{0} \tilde{\lambda}_{0},
$$

where $y$ is an arbitrary spinor satisfying $\langle y 0\rangle \neq 0$. One can verify that these satisfy the conditions in eq. (3.2). We can decompose the shifted momenta into spinors: $\hat{p}_{0} \sigma=\lambda_{0}^{z} \tilde{\lambda}_{0}$, $\hat{p}_{j} \sigma=\chi_{j}^{J} \tilde{\chi}_{j J}^{z}, \hat{p}_{k} \sigma=\chi_{k}^{J} \tilde{\chi}_{k J}^{z}$. At the spinor level, the shift is realized as ${ }^{6}$

$$
\begin{aligned}
\{\mathbf{0} \overline{\mathbf{m}} \overline{\mathbf{m}}\}: & \lambda_{0}^{z}=\epsilon \lambda_{0}-z y, \\
\tilde{\chi}_{j}^{z} & =\tilde{\chi}_{j}+\frac{(\epsilon-1)\left\langle 0 p_{k} 0\right]-z\left\langle y p_{k} 0\right]}{\left[0 p_{j} p_{k} 0\right]}[\mathbf{j} 0] \tilde{\lambda}_{0}, \\
\tilde{\chi}_{k}^{z} & =\tilde{\chi}_{k}-\frac{(\epsilon-1)\left\langle 0 p_{j} 0\right]-z\left\langle y p_{j} 0\right]}{\left[0 p_{j} p_{k} 0\right]}[\mathbf{k} 0] \tilde{\lambda}_{0},
\end{aligned}
$$

\footnotetext{
${ }^{6}$ In a different context, single parameter shifts of massive spinors were previously considered in refs. [32, $42]$.
} 
while all other spinors remain unshifted. Since we shift the holomorphic spinor corresponding to the particle 0 and the antiholomorphic spinors corresponding to the particles $j, k$, we label this shift as $\{0 \bar{m} \bar{m}\}$. The parity-reversed version, labelled as $\{\overline{0} m m\}$, is defined in appendix A. Note that our shift is non-trivial for $z=\epsilon=0$; in this limit the spinors $\tilde{\chi}_{j, k}$ must shift to absorb the original momentum of the particle 0 . This "zero-order" shift will play some role in the following. On the other hand, the shift is trivial for $z=0$ and $\epsilon=1$, but that is away from the soft limit $\epsilon \rightarrow 0$ on which we are focused in this paper.

We end this section with a number of identities that will be useful in the following (impatient readers are encouraged to skip to the next section). For the $\{0 \bar{m} \bar{m}\}$ shift, the location of the poles in eq. (3.4) can be recast as

$$
z_{l}=\epsilon \frac{\left\langle 0 p_{l} 0\right]}{\left\langle y p_{l} 0\right]} ; \quad m_{l}=0 \Rightarrow z_{l}=\epsilon \frac{\langle 0 l\rangle}{\langle y l\rangle} .
$$

At the poles, the shifted spinors can be expressed as

$$
\begin{aligned}
& \lambda_{0}^{z_{l}}=\epsilon \frac{\langle y 0\rangle}{\left\langle y p_{l} 0\right]} p_{l} \sigma \tilde{\lambda}_{0} \\
& \tilde{\chi}_{j}^{z_{l}}=\tilde{\chi}_{j}-\frac{2 p_{0} p_{k}}{\left[0 p_{j} p_{k} 0\right]}[\mathbf{j} 0] \tilde{\lambda}_{0}+\epsilon \frac{\langle y 0\rangle\left[0 p_{k} p_{l} 0\right]}{\left\langle y p_{l} 0\right\rangle\left[0 p_{j} p_{k} 0\right]}[\mathbf{j} 0] \tilde{\lambda}_{0}, \\
& \tilde{\chi}_{k}^{z_{l}}=\tilde{\chi}_{k}+\frac{2 p_{0} p_{j}}{\left[0 p_{j} p_{k} 0\right]}[\mathbf{k} 0] \tilde{\lambda}_{0}-\epsilon \frac{\langle y 0\rangle\left[0 p_{j} p_{l} 0\right]}{\left\langle y p_{l} 0\right\rangle\left[0 p_{j} p_{k} 0\right]}[\mathbf{k} 0] \tilde{\lambda}_{0} .
\end{aligned}
$$

These identities allow us to rewrite $\hat{P}_{0 l}$ at the poles. For $l \neq j, k, \hat{P}_{0 l}=\hat{p}_{0}+p_{l}$, thus

$$
\left.\hat{P}_{0 l} \sigma\right|_{z_{l}}=p_{l} \sigma+\epsilon \frac{\langle y 0\rangle}{\left\langle y p_{l} 0\right]} p_{l} \sigma \tilde{\lambda}_{0} \tilde{\lambda}_{0}, \quad l \neq j, k .
$$

Since $\left.\hat{P}_{0 l}\right|_{z=z_{l}}$ is on shell by definition, we can factorize it into spinors:

$$
\begin{aligned}
& m_{l}>\left.0 \Rightarrow \hat{P}_{0 l} \sigma\right|_{z_{l}}=\chi_{0 l}^{L} \tilde{\chi}_{0 l}, \quad \chi_{0 l}=\chi_{l}, \quad \tilde{\chi}_{0 l}=\tilde{\chi}_{l}+\epsilon \frac{\langle y 0\rangle}{\left\langle y p_{l} 0\right]}[10] \tilde{\lambda}_{0}, \quad l \neq j, k, \\
& m_{l}=\left.0 \Rightarrow \hat{P}_{0 l} \sigma\right|_{z_{l}}=\lambda_{0 l} \tilde{\lambda}_{0 l}, \quad \lambda_{0 l}=\lambda_{l}, \quad \tilde{\lambda}_{0 l}=\tilde{\lambda}_{l}+\epsilon \frac{\langle y 0\rangle}{\langle y l\rangle} \tilde{\lambda}_{0}, \quad l \neq j, k .
\end{aligned}
$$

Similarly at $z_{j}$ and $z_{k}$ we have the decomposition

$$
\begin{aligned}
& \left.\hat{P}_{0 j} \sigma\right|_{z_{j}}=p_{j} \sigma-\frac{2 p_{0} p_{k}}{\left[0 p_{j} p_{k} 0\right]} p_{j} \sigma \tilde{\lambda}_{0} \tilde{\lambda}_{0} \quad \Rightarrow \quad \chi_{0 j}=\chi_{j}, \quad \tilde{\chi}_{0 j}=\tilde{\chi}_{j}-\frac{2 p_{0} p_{k}}{\left[0 p_{j} p_{k} 0\right]}[\mathbf{j} 0] \tilde{\lambda}_{0}, \\
& \left.\hat{P}_{0 k} \sigma\right|_{z_{k}}=p_{k} \sigma+\frac{2 p_{0} p_{j}}{\left[0 p_{j} p_{k} 0\right]} p_{k} \sigma \tilde{\lambda}_{0} \tilde{\lambda}_{0}, \quad \Rightarrow \quad \chi_{0 k}=\chi_{k}, \quad \tilde{\chi}_{0 k}=\tilde{\chi}_{k}+\frac{2 p_{0} p_{j}}{\left[0 p_{j} p_{k} 0\right]}[\mathbf{k} 0] \tilde{\lambda}_{0} .
\end{aligned}
$$

In our story it will be important that these are independent of the soft parameter $\epsilon$. Similarly, the shifts $\tilde{\chi}_{j}^{z_{k}}$ and $\tilde{\chi}_{k}^{z_{j}}$ and are independent of $\epsilon$.

The case where one of the $j, k$ particles is massive and the other is massless is covered in appendix A. We also comment on the limit where all involved particles are massless. This can be easily obtained from eq. (3.9) by "unbolding" the massive spinors, which 
corresponds to replacing $\chi_{i}^{J} \rightarrow \lambda_{i}, \tilde{\chi}_{i}^{J} \rightarrow \tilde{\lambda}_{i}$ while dropping the $\mathrm{SU}(2)$ little group index. Simplifying the resulting expression one obtains

$$
\begin{aligned}
\{\mathbf{0} \overline{\mathbf{0}}\}: & \lambda_{0}^{z}=\epsilon \lambda_{0}-z y, \\
\tilde{\lambda}_{j}^{z} & =\tilde{\lambda}_{j}-\frac{(\epsilon-1)\langle 0 k\rangle-z\langle y k\rangle}{\langle j k\rangle} \tilde{\lambda}_{0}, \\
\tilde{\lambda}_{k}^{z} & =\tilde{\lambda}_{k}+\frac{(\epsilon-1)\langle 0 j\rangle-z\langle y j\rangle}{\langle j k\rangle} \tilde{\lambda}_{0},
\end{aligned}
$$

This reproduces the shift in ref. [16] up to the replacement $\epsilon \rightarrow \epsilon-1$ which is due to the different prescription for taking the soft limit.

\section{Soft theorems}

In this section we apply the recursion formula in eq. (3.7) to derive the soft factors for amplitudes containing particles of any mass and spin. This generalizes the discussion in ref. [16]. To our knowledge, this is the first derivation using the on-shell methods with massive spinors. The soft factors that we obtain are applicable to more general physical situations than those found in the previous literature. In the following we will deal with the soft particle of spin 2 (graviton) and spin 1 (photon), however eq. (3.7) goes just as well with massless particles of spin 0 (Goldstone bosons), spin 1/2 (Goldstone fermions), or spin $3 / 2$ (gravitinos). We restrict to single soft particle emission, leaving multiple soft limits for future publications.

\subsection{Minimal gravity}

We begin with a gravity theory where the massless spin-2 graviton is minimally coupled to matter and to itself. The soft theorems in this case have been studied back and forth, both in the standard Lagrangian and in the on-shell formalisms. Nevertheless, here we provide the soft theorems in a novel form that will be particularly convenient for practical calculations with massive spinors.

In the on-shell formalism, the minimal coupling of the graviton $h$ to a matter particle $X$ of mass $m>0$ and any spin $S$ corresponds to the on-shell 3-point amplitudes [22]

$$
\mathcal{M}\left(\mathbf{1}_{X} \mathbf{2}_{X} 3_{h}^{-}\right)=-\frac{1}{M_{\mathrm{Pl}}} \frac{\left\langle 3 p_{1} \zeta\right]^{2}}{[3 \zeta]^{2}} \frac{[\mathbf{2 1}]^{2 S}}{m^{2 S}}, \quad \mathcal{M}\left(\mathbf{1}_{X} \mathbf{2}_{X} 3_{h}^{+}\right)=-\frac{1}{M_{\mathrm{Pl}}} \frac{\left\langle\zeta p_{1} 3\right]^{2}}{\langle 3 \zeta\rangle^{2}} \frac{\langle\mathbf{2 1}\rangle^{2 S}}{m^{2 S}}
$$

where $M_{\mathrm{Pl}}=(8 \pi G)^{-1 / 2} \approx 2.4 \times 10^{18} \mathrm{GeV}$ is the Planck constant, and $\zeta, \tilde{\zeta}$ are arbitrary reference spinors satisfying $[3 \zeta] \neq 0$ and $\langle 3 \zeta\rangle \neq 0$ (the amplitude is independent of their precise choice). In the standard QFT language, the on-shell amplitude in eq. (4.1) corresponds to graviton coupling to matter via the energy-momentum tensor. Meanwhile, the minimal self-coupling of the graviton in the standard Einstein-Hilbert Lagrangian corresponds in the on-shell language to

$$
\mathcal{M}\left(1_{h}^{-} 2_{h}^{-} 3_{h}^{+}\right)=-\frac{1}{M_{\mathrm{Pl}}} \frac{\langle 12\rangle^{6}}{\langle 13\rangle^{2}\langle 23\rangle^{2}}, \quad \mathcal{M}\left(1_{h}^{+} 2_{h}^{+} 3_{h}^{-}\right)=-\frac{1}{M_{\mathrm{Pl}}} \frac{[12]^{6}}{[13]^{2}[23]^{2}} .
$$


We focus on emission of a soft graviton with plus helicity. The starting point is the recursion in eq. (3.7), with the amplitudes deformed by the $\{0 \bar{m} \bar{m}\}$ spinor shift in eq. (3.9). We first need an expression for the shifted 3-point amplitude at the $z_{l}$-poles:

$$
\hat{\mathcal{M}}^{z_{l}, \epsilon}\left(\left(-P_{0 l}\right) l 0_{h}^{+}\right)=-\frac{1}{M_{\mathrm{Pl}}} \frac{\left\langle y p_{l} 0\right]^{2}}{\epsilon^{2}\langle 0 y\rangle^{2}} .
$$

This expression is independent of the identity, mass, or spin of the $l$-th particle (except that for massive particles the identity operator in the little group indices is implicit), which is a realization of the equivalence principle. Plugging this back into eq. (3.7) we obtain a raw version of the soft theorem:

$$
\hat{\mathcal{M}}^{0, \epsilon}\left(1 \ldots n 0_{h}^{+}\right)=\frac{1}{\epsilon^{3} M_{\mathrm{Pl}}} \sum_{l=1}^{n} \frac{\left\langle y p_{l} 0\right]^{2}}{\left(2 p_{0} p_{l}\right)\langle 0 y\rangle^{2}} \hat{\mathcal{M}}^{z_{l}, \epsilon}\left(1 \ldots P_{0 l} \ldots n\right)+\mathcal{O}\left(\epsilon^{0}\right) .
$$

To proceed we need to expand the $n$-point amplitude above in powers of $\epsilon$. Using the identities in eq. (3.11) we find

$$
\hat{\mathcal{M}}^{z_{l}, \epsilon}\left(1 \ldots P_{0 l} \ldots n\right)=\left\{1+\epsilon \frac{\langle 0 y\rangle}{\left\langle y p_{l} 0\right]} \tilde{\mathcal{D}}_{l}+\frac{\epsilon^{2}}{2} \frac{\langle 0 y\rangle^{2}}{\left\langle y p_{l} 0\right]^{2}} \tilde{\mathcal{D}}_{l}^{2}\right\} \hat{\mathcal{M}}^{0,0}(1 \ldots n)+\mathcal{O}\left(\epsilon^{3}\right)
$$

where the differential operator $\tilde{\mathcal{D}}_{l}$ is

$$
\tilde{\mathcal{D}}_{l}=[0 \mathbf{l}]\left[0 \partial_{\mathbf{l}}\right]+\frac{\left[0 p_{k} p_{l} 0\right]}{\left[0 p_{j} p_{k} 0\right]}[0 \mathbf{j}]\left[0 \partial_{\mathbf{j}}\right]-\frac{\left[0 p_{j} p_{l} 0\right]}{\left[0 p_{j} p_{k} 0\right]}[0 \mathbf{k}]\left[0 \partial_{\mathbf{k}}\right], \quad l \neq j, k,
$$

and $\tilde{\mathcal{D}}_{l}=0$ for $l=j, k$. Here, $[0 \mathbf{l}]\left[0 \partial_{1}\right] \equiv\left(\tilde{\lambda}_{0} \tilde{\chi}_{l}^{L}\right)\left(\tilde{\lambda}_{0} \frac{\partial}{\partial \tilde{\chi}_{l}^{L}}\right)$ for a massive particle $l$, where for once we explicitly displayed the $\mathrm{SU}(2)$ little group index $L$, which is implicitly summed over. For a massless particle $l$ one should instead use $[0 l]\left[0 \partial_{l}\right] \equiv\left(\tilde{\lambda}_{0} \tilde{\lambda}_{l}\right)\left(\tilde{\lambda}_{0} \frac{\partial}{\partial \tilde{\lambda}_{l}}\right)$. Plugging the expanded $\hat{\mathcal{M}}^{z_{l}, \epsilon}$ back into eq. (4.4) we obtain the soft theorem in the standard form:

$$
\hat{\mathcal{M}}^{0, \epsilon}\left(1 \ldots n 0_{h}^{+}\right)=\left\{\frac{1}{\epsilon^{3}} S_{+2}^{(0)}+\frac{1}{\epsilon^{2}} S_{+2}^{(1)}+\frac{1}{\epsilon} S_{+2}^{(0)}\right\} \hat{\mathcal{M}}^{0,0}(1 \ldots n)+\mathcal{O}\left(\epsilon^{0}\right) .
$$

The leading soft factor is given by

$$
S_{+2}^{(0)}=\frac{1}{M_{\mathrm{Pl}}} \sum_{l=1}^{n} \frac{\left\langle y p_{l} 0\right]^{2}}{\left(2 p_{0} p_{l}\right)\langle 0 y\rangle^{2}}=\frac{1}{M_{\mathrm{Pl}}} \epsilon_{\mu \nu}^{0+} \sum_{l=1}^{n} \frac{p_{l}^{\mu} p_{l}^{\nu}}{p_{0} p_{l}} .
$$

In the second we step we rewrote the soft factor in the more familiar form as it appears in ref. [2], with the polarization tensor of the soft graviton defined below eq. (2.5) and the gauge parameter $\zeta$ is identified with the shift spinor $y$. This leading soft factor is independent of $y$ as a consequence of the equivalence principle and momentum conservation [43]:

$$
\frac{\partial}{\partial y} S_{+2}^{(0)} \sim \sum_{l=1}^{n} \frac{\left\langle y p_{l} 0\right]}{\langle y 0\rangle^{3}} y=-\frac{\left\langle y p_{0} 0\right]}{\langle y 0\rangle^{3}} y=0 .
$$


Conversely, the condition that $S_{+2}^{(0)}$ must be independent of the shift spinor $y$ can be employed to prove the equivalence principle without any reference to general coordinate invariance. ${ }^{7}$ Indeed, in our derivation, the soft theorems are a direct consequence of Poincaré invariance, locality, and unitarity. In a consistent theory they must lead to unambiguous soft factors for any choice of $y$. However, that would not be the case if the graviton coupling strength to matter in eq. (4.1) were not universal and precisely correlated with the graviton self-coupling in eq. (4.2).

The subleading soft factor in eq. (4.7) is a differential operator:

$$
S_{+2}^{(1)}=\frac{1}{M_{\mathrm{Pl}}} \sum_{l=1}^{n} \frac{\left\langle y p_{l} 0\right]}{\left(2 p_{0} p_{l}\right)\langle 0 y\rangle} \tilde{\mathcal{D}}_{l} .
$$

It is independent of the shift spinor $y$ as a consequence of angular momentum conservation. Indeed,

$$
\frac{\partial}{\partial y} S_{+2}^{(1)} \sim \sum_{l=1}^{n} \frac{\langle y 0\rangle p_{l} \sigma \tilde{\lambda}_{0}-\left\langle y p_{l} 0\right] \lambda_{0}}{\left(2 p_{0} p_{l}\right)\langle y 0\rangle^{2}} \tilde{\mathcal{D}}_{l}=-\sum_{l=1}^{n} \frac{\left\langle 0 p_{l} 0\right] y}{\left(2 p_{0} p_{l}\right)\langle y 0\rangle^{2}} \tilde{\mathcal{D}}_{l}=-\frac{y}{\langle y 0\rangle^{2}} \sum_{l=1}^{n} \tilde{\mathcal{D}}_{l} .
$$

Now, $\sum_{l=1}^{n} \tilde{\mathcal{D}}_{l}$ can be related to the angular momentum operator $J^{\mu \nu}$ introduced in [44] and generalized to massive particles in [24]:

$$
\begin{aligned}
J_{l}^{\mu \nu} & =J_{\chi_{l}}^{\mu \nu}+J_{\tilde{\chi}_{l}}^{\mu \nu}, \\
J_{\chi_{l}}^{\mu \nu} & \equiv \frac{i}{4}\left(\chi_{l} \sigma^{\mu} \bar{\sigma}^{\nu} \frac{\partial}{\partial \chi_{l}}-\chi_{l} \sigma^{\nu} \bar{\sigma}^{\mu} \frac{\partial}{\partial \chi_{l}}\right), \quad J_{\tilde{\chi}_{l}}^{\mu \nu} \equiv \frac{i}{4}\left(\tilde{\chi}_{l} \bar{\sigma}^{\mu} \sigma^{\nu} \frac{\partial}{\partial \tilde{\chi}_{l}}-\tilde{\chi}_{l} \bar{\sigma}^{\nu} \sigma^{\mu} \frac{\partial}{\partial \tilde{\chi}_{l}}\right) .
\end{aligned}
$$

For a massless particle $l$ one should replace $\chi \rightarrow \lambda$. Trading $\tilde{\mathcal{D}}_{l}$ for $J^{\mu \nu}$ we find

$$
\frac{\partial}{\partial y} S_{+2}^{(1)} \sim i \frac{\left(y \sigma^{\mu} \tilde{\lambda}_{0}\right) y}{\langle y 0\rangle^{3}} p_{0}^{\nu} \sum_{l=0}^{n} J_{l}^{\mu \nu}=0
$$

Finally, the sub-subleading soft factor in eq. (4.7) is a double-differential operator:

$$
S_{+2}^{(2)}=\frac{1}{4 M_{\mathrm{Pl}}} \sum_{l=1}^{n} \frac{1}{p_{0} p_{l}} \tilde{\mathcal{D}}_{l}^{2}
$$

This one is manifestly independent of the shift spinor $y$.

Following the same steps, but starting instead with the $\{\overline{0} m m\}$ shift defined in eq. (A.12), one can obtain the soft factors for emission of a minus helicity graviton:

$$
S_{-2}^{(0)}=\frac{1}{M_{\mathrm{Pl}}} \sum_{l=1}^{n} \frac{\left\langle 0 p_{l} y\right]^{2}}{\left(2 p_{0} p_{l}\right)[0 y]^{2}}, \quad S_{-2}^{(1)}=\frac{1}{M_{\mathrm{Pl}}} \sum_{l=1}^{n} \frac{\left\langle 0 p_{l} y\right]}{\left(2 p_{0} p_{l}\right)[0 y]} \mathcal{D}_{l}, \quad S_{-2}^{(2)}=\frac{1}{4 M_{\mathrm{Pl}}} \sum_{l=1}^{n} \frac{1}{p_{0} p_{l}} \mathcal{D}_{l}^{2},
$$

\footnotetext{
${ }^{7}$ One might however argue that, in the on-shell approach to soft theorems, the role of the shift spinor $y$ is exactly the same as that of the gauge parameter in the standard approach. From this point of view, gauge invariance thrown out of the door, comes back in through the window. On the other hand, ref. [16] derives the same conclusion by demanding the absence of double poles in $z$, in which case the parallel with gauge invariance is avoided.
} 
where

$$
\mathcal{D}_{l}=\langle 0 \mathbf{l}\rangle\left\langle 0 \partial_{\mathbf{l}}\right\rangle+\frac{\left\langle 0 p_{k} p_{l} 0\right\rangle}{\left\langle 0 p_{j} p_{k} 0\right\rangle}\langle 0 \mathbf{j}\rangle\left\langle 0 \partial_{\mathbf{j}}\right\rangle-\frac{\left\langle 0 p_{j} p_{l} 0\right\rangle}{\left\langle 0 p_{j} p_{k} 0\right\rangle}\langle 0 \mathbf{k}\rangle\left\langle 0 \partial_{\mathbf{k}}\right\rangle, \quad l \neq j, k \text { and } \mathcal{D}_{l}=0, \quad l=j, k .
$$

Let us briefly comment on the relation of our results with the previous literature. It is a simple exercise to show that in the limit where all particles (also $j$ and $k$ ) are massless, the soft factors we derived reduce to those found in [16]. In particular, the differential operator $\tilde{\mathcal{D}}_{l}$ reduces to

$$
\tilde{\mathcal{D}}_{l} \rightarrow[0 l]\left\{\left[0 \partial_{l}\right]-\frac{\langle l k\rangle}{\langle j k\rangle}\left[0 \partial_{j}\right]+\frac{\langle l j\rangle}{\langle j k\rangle}\left[0 \partial_{k}\right]\right\}=[0 l] \nabla_{0 l},
$$

where $\nabla_{0 l}$ is defined in eq. (19) of [16]. On the other hand, our soft theorems do not exactly reduce to those in [11], the obstruction being the last two terms in the curly bracket in eq. (4.17). As discussed in [16], this difference is due to working with "stripped" amplitudes $\mathcal{M}$, rather than with $S$ matrix elements $T \sim \delta^{4}\left(p_{0}+\cdots+p_{N}\right) \mathcal{M}$ as in [11]. The price to pay for us is that the soft theorems look somewhat more complicated, and that they depend on the exact manner in which the soft limit is taken. The advantage is that for practical purposes one works with the stripped amplitudes $\mathcal{M}$. The expressions we provide are ready to use out of the box with massive amplitudes written using the modern on-shell spinor formalism.

\subsection{Non-minimal gravity}

We move to consider theories where gravitational interactions of matter are non-minimal. Let us start by assuming that a massive particle $X$ with spin $S>0$ and mass $m>0$ has an anomalous gravitomagnetic dipole moment:

$$
\mathcal{M}\left(\mathbf{1}_{X} \mathbf{2}_{X} 3_{h}^{+}\right) \stackrel{?}{=}-\frac{1}{M_{\mathrm{Pl}} m^{2 S}} \frac{\left\langle\zeta p_{1} 3\right]^{2}}{\langle 3 \zeta\rangle^{2}}\left[\langle\mathbf{2 1}\rangle^{2 S}+\frac{d_{X}}{m^{2}} \frac{\left\langle\zeta p_{1} 3\right]}{\langle 3 \zeta\rangle}\langle 3 \mathbf{1}\rangle\langle 3 \mathbf{2}\rangle\langle\mathbf{2 1}\rangle^{2 S-1}\right] .
$$

It was argued in ref. [34] that general coordinate invariance forbids $d_{X} \neq 0$. But let us press on to see what happens. It turns out that the dipole affects the subleading soft factor, which is modified as

$$
S_{+2}^{(1)}=\frac{1}{M_{\mathrm{Pl}}} \sum_{l=1}^{n} \frac{\left\langle y p_{l} 0\right]}{\left(2 p_{0} p_{l}\right)\langle 0 y\rangle}\left[\delta_{\vec{L}}^{\vec{J}} \tilde{\mathcal{D}}_{l}+\frac{d_{l}}{m_{l}^{2}} \delta_{L_{2} \ldots L_{2 S_{l}}}^{J_{2} \ldots J_{2 S_{l}}}\left(\tilde{\chi}_{l}^{J_{1}} \tilde{\lambda}_{0}\right)\left(\tilde{\chi}_{l L_{1}} \tilde{\lambda}_{0}\right)\right] .
$$

The first term in the bracket comes from the minimal coupling, and is the same as in eq. (4.10), except this time we explicitly display the identity operator acting on the little group indices of the $l$-th particle. The second factor is due to the dipole, where, as always, symmetrization of $J_{1} \ldots J_{2 S_{l}}$ and $L_{1} \ldots L_{2 S_{l}}$ is implicit. The problem is that, for $d_{l} \neq 0$, this soft factor is not independent of the shift spinor:

$$
\frac{\partial}{\partial y} S_{+2}^{(1)} \sim \frac{y}{\langle y 0\rangle^{2}} \delta_{L_{2} \ldots L_{2 S_{l}}}^{J_{2} \ldots J_{2}} \sum_{l=1}^{n} \frac{d_{l}}{m_{l}^{2}}\left(\tilde{\chi}_{l}^{J_{1}} \tilde{\lambda}_{0}\right)\left(\tilde{\chi}_{l L_{1}} \tilde{\lambda}_{0}\right) \neq 0 .
$$

This provides a simple and general proof that, for a particle of any mass and spin, the presence of an anomalous gravitomagnetic dipole moment is in conflict with the assumptions of Poincaré invariance, locality and unitarity. 
Given the dipole is forbidden, for $S \geq 1$ the leading deformation of the minimal coupling is the quadrupole:

$$
\begin{aligned}
& \mathcal{M}\left(\mathbf{1}_{X} \mathbf{2}_{X} 3_{h}^{+}\right)=-\frac{1}{M_{\mathrm{Pl}} m^{2 S}} \frac{\left\langle\zeta p_{1} 3\right]^{2}}{\langle 3 \zeta\rangle^{2}}\left[\langle\mathbf{2 1}\rangle^{2 S}+\frac{Q}{2 m^{4}} \frac{\left\langle\zeta p_{1} 3\right]^{2}}{\langle 3 \zeta\rangle^{2}}\langle 3 \mathbf{1}\rangle^{2}\langle 3 \mathbf{2}\rangle^{2}\langle\mathbf{2 1}\rangle^{2 S-2}+\ldots\right], \\
& \mathcal{M}\left(\mathbf{1}_{X} \mathbf{2}_{X} 3_{h}^{-}\right)=-\frac{1}{M_{\mathrm{Pl}} m^{2 S}} \frac{\left\langle 3 p_{1} \zeta\right]^{2}}{[3 \zeta]^{2}}\left[[\mathbf{2 1}]^{2 S}+\frac{Q}{2 m^{4}} \frac{\left\langle 3 p_{1} \zeta\right]^{2}}{[3 \zeta]^{2}}[3 \mathbf{1}]^{2}[3 \mathbf{2}]^{2}[\mathbf{2 1}]^{2 S-2}+\ldots\right],(4
\end{aligned}
$$

where the dots stand for eventual higher multipoles, which do not affect the soft theorems. ${ }^{8}$ The quadrupole does arise from local Lagrangians, roughly speaking, when gravity interacts with matter via the Riemann tensor; for example, for $S=1$ it corresponds to the coupling $\mathcal{L} \sim Q X_{\mu \nu} X_{\alpha \beta} R_{\mu \nu \alpha \beta}$.

We find that the quadrupole enters into the sub-subleading soft factor:

$$
\begin{aligned}
& S_{+2}^{(2)}=\frac{1}{4 M_{\mathrm{Pl}}} \sum_{l=1}^{n} \frac{1}{p_{0} p_{l}}\left[\tilde{\mathcal{D}}_{l}^{2} \delta_{\vec{L}}^{\vec{J}}+\frac{Q_{l}}{m_{l}^{2}}\left(\tilde{\chi}_{l}^{J_{1}} \tilde{\lambda}_{0}\right)\left(\tilde{\chi}_{l}^{J_{2}} \tilde{\lambda}_{0}\right)\left(\tilde{\chi}_{l L_{1}} \tilde{\lambda}_{0}\right)\left(\tilde{\chi}_{l L_{2}} \tilde{\lambda}_{0}\right) \delta_{L_{3} \ldots L_{2 S_{l}}}^{J_{3} \ldots J_{2 S_{l}}}\right] \\
& S_{-}^{(2)}=\frac{1}{4 M_{\mathrm{Pl}}} \sum_{l=1}^{n} \frac{1}{p_{0} p_{l}}\left[\mathcal{D}_{l}^{2} \delta_{\vec{L}}^{\vec{J}}+\frac{Q_{l}}{m_{l}^{2}}\left(\chi_{l}^{J_{1}} \lambda_{0}\right)\left(\chi_{l}^{J_{2}} \lambda_{0}\right)\left(\chi_{l L_{1}} \lambda_{0}\right)\left(\chi_{l L_{2}} \lambda_{0}\right) \delta_{L_{3} \ldots L_{2 S_{l}}}^{J_{3} \ldots J_{2 S_{l}}}\right]
\end{aligned}
$$

where, as always, symmetrization over the little group indices is understood. The doubledifferential operator due to the minimal coupling is now supplemented by an algebraic operator originating from the quadrupole. Note that the latter acts non-trivially on the little group indices: the $l$-th term in the sum acts on the upper indices $\vec{L} \equiv L_{1} \ldots L_{2 S_{l}}$ of the $l$-th particle in $\hat{\mathcal{M}}^{0,0}\left(1 \ldots l^{\vec{L}} \ldots n\right)$.

\subsection{Photons}

Photons can be tackled by exactly the same methods as gravitons, except that the calculations are simpler. Below we summarize the soft theorems for photon emission. Electromagnetic interactions of a particle $X$ with mass $m$ and spin $S$ are described by the following 3 -point amplitudes [22, 34]:

$$
\begin{aligned}
& \mathcal{M}\left(\mathbf{1}_{X} \mathbf{2}_{\bar{X}} 3_{\gamma}^{+}\right)=\frac{\sqrt{2} e}{m^{2 S}}\left\{q \frac{\left\langle\zeta p_{1} 3\right]}{\langle 3 \zeta\rangle}\langle\mathbf{2 1}\rangle^{2 S}+\frac{S a}{m^{2}} \frac{\left\langle\zeta p_{1} 3\right]^{2}}{\langle 3 \zeta\rangle^{2}}\langle 3 \mathbf{1}\rangle\langle 3 \mathbf{2}\rangle\langle\mathbf{2 1}\rangle^{2 S-1}+\ldots\right\}, \\
& \mathcal{M}\left(\mathbf{1}_{X} \mathbf{2}_{\bar{X}} 3_{\gamma}^{-}\right)=\frac{\sqrt{2} e}{m^{2 S}}\left\{q \frac{\left\langle 3 p_{1} \zeta\right]}{[3 \zeta]}[\mathbf{2 1}]^{2 S}+\frac{S \bar{a}}{m^{2}} \frac{\left\langle 3 p_{1} \zeta\right]^{2}}{[3 \zeta]^{2}}[3 \mathbf{1}][3 \mathbf{2}][\mathbf{2 1}]^{2 S-1}+\ldots\right\},
\end{aligned}
$$

where $e$ is the electromagnetic coupling constant, $q$ is the electric charge, $(a+\bar{a}) / 2=$ $(g-2) / 2$ is the anomalous magnetic moment in the standard normalization, $a \neq \bar{a}$ gives the electric dipole moment, and the dots stand for eventual higher multipoles which are irrelevant for the soft theorems. The first term in each bracket describes the minimal coupling, while the second describes non-minimal dipole interactions for $S \geq 1 / 2$. In the

\footnotetext{
${ }^{8}$ The $n$-th multipole enters the recursion in eq. (3.7) with the $\frac{\left(\lambda_{0}^{z_{l}} \chi_{1}\right)^{n}\left(\lambda_{0}^{z_{l}} \chi_{2}\right)^{n}}{\left(\lambda_{0}^{z_{l}} \zeta\right)^{n}}$ factor, which scales as $\epsilon^{n}$, given $\lambda_{0}^{z_{l}} \sim \epsilon$.
} 
Lagrangian parlance, the former arises when photon interacts via $D_{\mu}=\partial_{\mu}-i e q A_{\mu}$ in the kinetic term, while the higher multipoles correspond to photon interacting via $F_{\mu \nu}$. For example, for real $a$, the $S=1 / 2$ Lagrangian would be $\mathcal{L} \supset e q \bar{\psi} \gamma_{\mu} \psi A_{\mu}+\frac{e a}{4 m} \bar{\psi} \sigma_{\mu \nu} \psi F^{\mu \nu}$, while for $S=1$ it would be $\mathcal{L} \supset-i q e\left(V_{\mu \nu} \bar{V}_{\nu}-\bar{V}_{\mu \nu} V_{\nu}\right) A_{\mu}+i e(q+2 a) V_{\mu} \bar{V}_{\nu} F_{\mu \nu}$.

Plugging the plus helicity amplitude in eq. (4.23) into the recursion in eq. (3.7) one finds the raw version of the soft theorem:

$$
\begin{aligned}
\hat{\mathcal{M}}^{0, \epsilon}\left(1 \ldots n 0^{+}\right)= & -\frac{e}{\sqrt{2}} \sum_{l=1}^{n} \frac{1}{p_{0} p_{l}}\left[\frac{q_{l}\left\langle y p_{l} 0\right]}{\epsilon^{2}\langle 0 y\rangle} \delta_{\vec{L}}^{\vec{J}}+\frac{S_{l} a_{l}}{m_{l} \epsilon}\left(\tilde{\lambda}_{0} \tilde{\chi}_{l}^{J_{1}}\right)\left(\tilde{\lambda}_{0} \tilde{\chi}_{l L_{1}}\right) \delta_{L_{2} \ldots L_{2 S_{l}}}^{J_{2} \ldots J_{2 S_{l}}}\right] \hat{\mathcal{M}}^{z_{l}, \epsilon}\left(1 \ldots P_{0 l}^{\vec{L}} \ldots n\right) \\
& +\mathcal{O}\left(\epsilon^{0}\right) .
\end{aligned}
$$

The first term in the bracket originates from the minimal coupling, while the second describes a correction from the dipole. The latter has a non-trivial action on the little group indices of $\hat{\mathcal{M}}$. Using the expansion of $\hat{\mathcal{M}}$ in powers of $\epsilon$, cf. eq. (4.5), one derives the soft theorem:

$$
\hat{\mathcal{M}}^{0, \epsilon}\left(1 \ldots n 0^{ \pm}\right)=-\left\{\frac{1}{\epsilon^{2}} S_{ \pm 1}^{(0)}+\frac{1}{\epsilon} S_{ \pm 1}^{(1)}\right\} \hat{\mathcal{M}}^{0,0}(1 \ldots n)+\mathcal{O}\left(\epsilon^{0}\right) .
$$

The leading soft factor for emission of a plus helicity photon is

$$
S_{+1}^{(0)}=\frac{e}{\sqrt{2}} \sum_{l=1}^{n} \frac{q_{l}\left\langle y p_{l} 0\right]}{\langle 0 y\rangle\left(p_{0} p_{l}\right)}=e \epsilon_{\mu}^{0+} \sum_{l=1}^{n} \frac{q_{l} p_{l}^{\mu}}{p_{0} p_{l}},
$$

where in the second step we introduced the polarization vector of the soft photon defined in eq. (2.5), so as to make contact with the Weinberg's formula. The independence of $S^{(0)}$ on the shift spinor $y$ follows from charge conservation [43]:

$$
\frac{\partial}{\partial y} S_{+1}^{(0)} \sim \frac{y}{\langle y 0\rangle^{2}} \sum_{l=1}^{n} q_{l}=0
$$

or the other way around: given that the soft recursion must be valid for any $y$ proves charge conservation in any Poincaré invariant, local, and unitary theory.

The subleading soft factor is given by

$$
S_{+1}^{(1)}=\frac{e}{\sqrt{2}} \sum_{l=1}^{n} \frac{1}{p_{0} p_{l}}\left[q_{l} \tilde{\mathcal{D}}_{l} \delta_{\vec{L}}^{\vec{J}}+\frac{S_{l} a_{l}}{m_{l}}\left(\tilde{\lambda}_{0} \tilde{\chi}_{l}^{J_{1}}\right)\left(\tilde{\lambda}_{0} \tilde{\chi}_{l L_{1}}\right) \delta_{L_{2} \ldots L_{2 S_{l}}}^{J_{2} \ldots J_{2 S_{l}}}\right],
$$

where $\tilde{\mathcal{D}}_{l}$ is defined in eq. (4.6). This one is manifestly independent of $y$, which in particular confirms what everybody knows that arbitrary electromagnetic dipoles are allowed. Here, we display the action on little group indices of a massive $l$-th particle, which is non-trivial when dipoles are involved. If on the other hand the $l$-th particle is massless, we should unbold the $l$-th term above, that is trade $\chi_{l}^{L} \rightarrow \lambda_{l}$, dropping the $\mathrm{SU}(2)$ little group indices. Finally, the soft factors for emission of a minus helicity photon are

$$
\begin{aligned}
S_{-1}^{(0)} & =\frac{e}{\sqrt{2}} \sum_{l=1}^{n} \frac{q_{l}\left\langle 0 p_{l} y\right]}{[0 y]\left(p_{0} p_{l}\right)}=e \epsilon_{\mu}^{0-} \sum_{l=1}^{n} \frac{q_{l} p_{l}^{\mu}}{p_{0} p_{l}}, \\
S_{-1}^{(1)} & =\frac{e}{\sqrt{2}} \sum_{l=1}^{n} \frac{1}{p_{0} p_{l}}\left[q_{l} \mathcal{D}_{l} \delta_{\vec{L}}^{\vec{J}}+\frac{S_{l} \bar{a}_{l}}{m_{l}}\left(\lambda_{0} \chi_{l}^{J_{1}}\right)\left(\lambda_{0} \chi_{l} L_{1}\right) \delta_{L_{2} \ldots L_{2 S_{l}}}^{J_{2} \ldots J_{2 S_{l}}}\right] .
\end{aligned}
$$




\section{Applications}

The goal of this section is to demonstrate that the recursion relations and soft theorems we obtained previously allow one to efficiently calculate amplitudes with massive particles of arbitrary spin. Below we will reproduce some known results, as well as derive new ones not encountered in the literature so far.

\subsection{Compton scattering}

We start with the Compton scattering. We are interested in the amplitude $\mathcal{M}\left(\mathbf{1}_{X} \mathbf{2}_{\bar{X}} 3_{\gamma}^{ \pm} 0_{\gamma}^{+}\right)$, where $0_{\gamma}$ is a soft photon ${ }^{9}$ of plus helicity, and $X$ is a particle of mass $m$, unit charge, and arbitrary spin $S$. We will use the $\{0 \bar{m} \bar{m}\}$ shift in eq. (3.9) with $j=1$ and $k=2$. We first assume minimal coupling, that is the absence of the dipole and higher multipoles in eq. (4.23) (the dipole case will be discussed shortly). The soft theorem in eq. (4.25) reduces to

$$
\hat{\mathcal{M}}^{0, \epsilon}\left(\mathbf{1}_{X} \mathbf{2}_{\bar{X}} 3_{\gamma}^{ \pm} 0_{\gamma}^{+}\right)=-\frac{1}{\epsilon^{2}} S_{+1}^{(0)} \hat{\mathcal{M}}^{0,0}\left(\mathbf{1}_{X} \mathbf{2}_{\bar{X}} 3_{\gamma}^{ \pm}\right)+B_{\infty}
$$

In this case the subleading soft factor $S_{+1}^{(1)}$ is void for the simple reason that, for Compton scattering, we only have two factorization poles $z_{1,2}$, and $\tilde{\mathcal{D}}_{l}$ in eq. (4.6) is zero for $l=j, k$. Somewhat pedantically, we replaced $\mathcal{O}\left(\epsilon^{0}\right)$ in eq. (4.25) by the boundary term $B_{\infty}$, which is also $\mathcal{O}\left(\epsilon^{0}\right)$. The point is that, for 4-body processes, photon emission from external legs accounts for all factorization poles. Therefore, the recursion relation in eq. (3.7) should reproduce the full Compton amplitude (not just the leading terms for $\epsilon \rightarrow 0$ ) up to the boundary term introduced in eq. (3.3). The leading soft factor for Compton scattering is easily calculated as

$$
S_{+1}^{(0)}=\sqrt{2} e \frac{\left\langle 3 p_{1} 0\right][03]}{\left(2 p_{1} p_{0}\right)\left(2 p_{2} p_{0}\right)} .
$$

For minimal coupling, the hard 3-point amplitude on the right-hand side of eq. (5.1) evaluates to

$$
\begin{aligned}
& \hat{\mathcal{M}}^{0,0}\left(\mathbf{1}_{X} \mathbf{2}_{\bar{X}} 3_{\gamma}^{-}\right)=\sqrt{2} e \frac{\left\langle 3 p_{1} 0\right]}{[30]}\left[-\frac{\langle\mathbf{1 3}\rangle[\mathbf{2} 0]+\langle\mathbf{2} 3\rangle[\mathbf{1 0} 0]}{\left\langle 3 p_{1} 0\right]}\right]^{2 S}, \\
& \hat{\mathcal{M}}^{0,0}\left(\mathbf{1}_{X} \mathbf{2}_{\bar{X}} 3_{\gamma}^{+}\right)=\sqrt{2} e \frac{[30]}{\left\langle 3 p_{1} 0\right]} \frac{\langle\mathbf{2 1}\rangle^{2 S}}{m^{2 S-2}}
\end{aligned}
$$

It is important to stress that the shift is not void in $\hat{\mathcal{M}}^{0,0}$. That is because the shift we defined in eq. (3.9) is non-trivial for $z=\epsilon=0$ (it is trivial if $\epsilon=1$ instead). For this reason $\hat{\mathcal{M}}^{0,0}\left(\mathbf{1}_{X} \mathbf{2}_{\bar{X}} 3_{\gamma}^{ \pm}\right)$above is not simply a copy of $\mathcal{M}\left(\mathbf{1}_{X} \mathbf{2}_{\bar{X}} 3_{\gamma}^{ \pm}\right)$in eq. (5.1). This zero-order shift is absolutely crucial to recover the correct result. In particular, the non-trivial spinor structure in $\hat{\mathcal{M}}^{0,0}\left(\mathbf{1}_{X} \mathbf{2}_{\bar{X}} 3_{\gamma}^{-}\right)$appears as a consequence of the identity

\footnotetext{
${ }^{9}$ In the Mandelstam variables the soft photon limit $p_{0} \rightarrow 0$ corresponds to $t \rightarrow m^{2}, u \rightarrow m^{2}, s \rightarrow 0$. For $m>0$ this of course cannot be realized with real kinematics, but the limit does make sense in complex kinematics.
} 
$\left.\left(\tilde{\chi}_{1}^{z} \tilde{\chi}_{2}^{z}\right)\right|_{z=\epsilon=0}=m \frac{\langle\mathbf{1 3}\rangle[\mathbf{2 0} 0]+\langle\mathbf{2 3}\rangle[\mathbf{1 0}]}{\left\langle 3 p_{1} 0\right]}$. All in all we reconstruct

$$
\begin{aligned}
& \hat{\mathcal{M}}^{0, \epsilon}\left(\mathbf{1}_{X} \mathbf{2}_{\bar{X}} 3_{\gamma}^{-} 0_{\gamma}^{+}\right)=\frac{2 e^{2}\left\langle 3 p_{1} 0\right]^{2}}{\epsilon^{2}\left(2 p_{1} p_{0}\right)\left(2 p_{2} p_{0}\right)}\left[-\frac{\langle\mathbf{1} 3\rangle[\mathbf{2} 0]+\langle\mathbf{2} 3\rangle[\mathbf{1 0} 0]}{\left\langle 3 p_{1} 0\right]}\right]^{2 S}+B_{\infty}, \\
& \hat{\mathcal{M}}^{0, \epsilon}\left(\mathbf{1}_{X} \mathbf{2}_{\bar{X}} 3_{\gamma}^{+} 0_{\gamma}^{+}\right)=\frac{2 e^{2}[30]^{2}}{\epsilon^{2}\left(2 p_{1} p_{0}\right)\left(2 p_{2} p_{0}\right)} \frac{\langle\mathbf{2 1}\rangle^{2 S}}{m^{2 S-2}}+B_{\infty}^{\prime} .
\end{aligned}
$$

On general grounds, this correctly describes leading terms of the Compton amplitude in the limit when the momentum of $0_{\gamma}^{+}$is soft. The full amplitude valid for any momentum of $0_{\gamma}^{+}$is recovered by setting $\epsilon=1$. In this limit, the same-helicity amplitude above is a fully consistent expression for any $S$, also when $B_{\infty}^{\prime}=0 .{ }^{10}$ A non-zero $B_{\infty}^{\prime}$ corresponds to a contact term (that is to say, without any singularities in the kinematic variables); for example we can have $B_{\infty}^{\prime}=\frac{[\mathbf{2 1}]^{2 S}}{m^{2 S}} \frac{[30]^{2}}{\Lambda^{2}}$. On the other hand, the opposite-helicity amplitude is healthy as it stands only for $S \leq 1$. As discussed in [22], for $S>1$ the first term in eq. (5.4) develops an unphysical pole at $\left\langle 3 p_{1} 0\right] \rightarrow 0$. That means the boundary term cannot vanish for $S>1$; it must be non-zero to cancel the unphysical pole, and at the same time it cannot have any singularities as $p_{0} p_{1} \rightarrow 0$ or $p_{0} p_{2} \rightarrow 0$. Therefore it must be of the form $B_{\infty}=\sum_{k=1}^{2 S-2} \frac{C_{k}}{\left\langle 3 p_{1} 0\right]^{k}}$, where $C_{k}$ are pure contact terms.

One possible form of the boundary term is

$$
\begin{aligned}
B_{\infty} & =-\frac{2 e^{2}}{\left(2 m^{2}\right)^{2 S}\left(2 p_{1} p_{0}\right)\left(2 p_{2} p_{0}\right)} \sum_{k=3}^{2 S}\left(\begin{array}{c}
2 S \\
k
\end{array}\right) \frac{\mathcal{Y}\left(\mathcal{Y}^{k-1}-\{\mathcal{Y} \| \mathcal{Z}\} \mathcal{Z}^{k-2}\right)(-\mathcal{X})^{2 S-k}}{\left\langle 3 p_{1} 0\right]^{k-2}} \\
\mathcal{Y} & \equiv\left(2 p_{1} p_{0}\right)\langle\mathbf{1 3}\rangle[\mathbf{2} 0]+\left(2 p_{2} p_{0}\right)\langle\mathbf{2 3}\rangle[\mathbf{1} 0], \quad \mathcal{Z} \equiv\left(2 p_{1} p_{0}\right)\langle\mathbf{1} 3\rangle[\mathbf{2} 0]-\left(2 p_{2} p_{0}\right)\langle\mathbf{2 3}\rangle[\mathbf{1} 0] \\
\mathcal{X} & \equiv m\langle\mathbf{1 2}\rangle+m[\mathbf{1 2}]-\left\langle\mathbf{1} p_{0} \mathbf{2}\right]-\left\langle\mathbf{2} p_{3} \mathbf{1}\right],
\end{aligned}
$$

where $\{\mathcal{Y} \| \mathcal{Z}\}=\mathcal{Y}$ for even $k$, and $\{\mathcal{Y} \| \mathcal{Z}\}=\mathcal{Z}$ for odd $k$. Any other legal boundary term will differ from the one above only by contact terms. The absence of physical poles in eq. (5.5) is not manifest, but it immediately follows from $\mathcal{Y}^{2 n}-\mathcal{Z}^{2 n}=\left(\mathcal{Y}^{2}-\mathcal{Z}^{2}\right)\left(\mathcal{Y}^{2 n-2}+\right.$ $\left.\cdots+\mathcal{Z}^{2 n-2}\right)$, given that $\left(\mathcal{Y}^{2}-\mathcal{Z}^{2}\right)=16\left(p_{1} p_{0}\right)\left(p_{2} p_{0}\right)\langle\mathbf{1 3}\rangle[\mathbf{2 0}]\langle\mathbf{2 3}\rangle[\mathbf{1 0}]$. A rationale for this boundary term is postponed to appendix B. For the present discussion we notice that $B_{\infty}$ is a polynomial in $z$ after applying our $\{0 \bar{m} \bar{m}\}$ shift, which is due to the fact that the shift enters only via the $p_{1} p_{0}$ and $p_{2} p_{0}$ factors in $\mathcal{Y}$ and $\mathcal{Z}$. More precisely, the boundary term is of the form $B_{\infty}=\sum_{n=1}^{2 S-2} \frac{P_{n}(z)}{\left\langle 3 p_{1} 0\right]^{n}}$, where $P_{n}(z)$ is the $n$-th order polynomial in $z$. Furthermore, $P_{n}(z) \sim \epsilon^{n}$ in the soft limit. Thus the boundary term does not show up in the soft theorems, but still it contributes to the recursion in eq. (3.7) via the integral over the circle at infinity. Overall $B_{\infty} \sim \mathcal{O}\left(\epsilon^{1}\right)$, better than $\mathcal{O}\left(\epsilon^{0}\right)$ deduced on general grounds. The boundary term we propose is arguably more compact than the one quoted in [34]. It also makes manifest that the UV behavior of Compton scattering for $S>1$ can be $\mathcal{O}\left(E^{4 S} / m^{4 S}\right)$. In fact, one can further soften it down to $\mathcal{O}\left(E^{4 S-2} / m^{4 S-2}\right)$ by subtracting from it a judiciously chosen contact term. See appendix B for more details.

We turn to another example where the subleading soft factor is no longer moot. We consider Compton scattering for arbitrary $S \geq 1 / 2$ when the dipole $a$ in eq. (4.23) is

\footnotetext{
${ }^{10}$ For $S>1$, the same-helicity Compton amplitude diverges as $\left(p_{1}+p_{2}\right)^{2} \rightarrow \infty$, in which case it is consistent in the sense of an effective theory amplitude below some cutoff scale.
} 
switched on. In order to keep this example simple, we restrict to the case where $q=0$, however a more general formula can be easily worked out by the same methods. The hard amplitude with a minus helicity photon is given by

$$
\hat{\mathcal{M}}^{0,0}\left(1_{X} 2_{\bar{X}} 3_{\gamma}^{-}\right)=(-1)^{2 S+1} \frac{\sqrt{2} e S \bar{a}}{m}\langle\mathbf{1 3}\rangle\langle\mathbf{2} 3\rangle\left[\frac{\langle\mathbf{1 3}\rangle[\mathbf{2} 0]+\langle\mathbf{2} 3\rangle[\mathbf{1 0} 0]}{\left\langle 3 p_{1} 0\right]}\right]^{2 S-1} .
$$

The subleading soft factor for a plus helicity photon takes the form

$$
S_{+1}^{(1)}=\frac{\sqrt{2} S e a}{m}\left[\frac{[0 \mathbf{1}]\left(\tilde{\lambda}_{0} \tilde{\chi}_{1 L_{1}}\right)}{2 p_{0} p_{1}}-\frac{[0 \mathbf{2}]\left(\tilde{\lambda}_{0} \tilde{\chi}_{2 K_{1}}\right)}{2 p_{0} p_{2}}\right] .
$$

Only those little group indices that act non-trivially on the hard factor are displayed. With a little help of spinor algebra one can show that this action amounts to

$$
\begin{aligned}
& {[0 \mathbf{1}]\left(\tilde{\lambda}_{0} \tilde{\chi}_{1 L_{1}}\right)\left\{\langle\mathbf{1 3}\rangle\langle\mathbf{2} 3\rangle\left(\langle\mathbf{1 3}\rangle[\mathbf{2} 0]+\langle\mathbf{2 3}\rangle[\mathbf{1 0} 0)^{2 S-1}\right\}\right.} \\
& =-\left\langle 3 p_{1} 0\right]\langle\mathbf{2} 3\rangle[\mathbf{1 0} 0](\langle\mathbf{1 3}\rangle[\mathbf{2 0}]+\langle\mathbf{2} 3\rangle[\mathbf{1 0} 0])^{2 S-2} \\
& \times\left[\langle\mathbf{1 3}\rangle[\mathbf{2 0}]+\frac{1}{2 S}\langle\mathbf{2} 3\rangle[\mathbf{1 0}]\right], \\
& {[0 \mathbf{2}]\left(\tilde{\lambda}_{0} \tilde{\chi}_{2 K_{1}}\right)\left\{\langle\mathbf{1 3}\rangle\langle\mathbf{2} 3\rangle(\langle\mathbf{1 3}\rangle[\mathbf{2 0}]+\langle\mathbf{2} 3\rangle[\mathbf{1 0}])^{2 S-1}\right\}} \\
& =\left\langle 3 p_{1} 0\right]\langle\mathbf{1 3}\rangle[\mathbf{2} 0](\langle\mathbf{1 3}\rangle[\mathbf{2} 0]+\langle\mathbf{2} 3\rangle[\mathbf{1 0}])^{2 S-2} \\
& \times\left[\frac{1}{2 S}\langle\mathbf{1 3}\rangle[\mathbf{2 0}]+\langle\mathbf{2} 3\rangle[\mathbf{1 0}]\right] .
\end{aligned}
$$

The $1 / 2 S$ factors appear as a consequence of symmetrization of the little group indices. Putting it all together:

$$
\begin{aligned}
\hat{\mathcal{M}}^{0, \epsilon}\left(\mathbf{1}_{X} \mathbf{2}_{\bar{X}} 3_{\gamma}^{-} 0_{\gamma}^{+}\right)= & -\frac{1}{\epsilon} S_{+1}^{(1)} \hat{\mathcal{M}}^{0,0}\left(1_{X} 2_{\bar{X}} 3_{\gamma}^{-}\right)+B_{\infty} \\
= & |a|^{2} \frac{(-1)^{2 S} S e^{2}}{\epsilon m^{2}\left(2 p_{1} p_{0}\right)\left(2 p_{2} p_{0}\right)}\left[\frac{\langle\mathbf{1 3}\rangle[\mathbf{2} 0]+\langle\mathbf{2} 3\rangle[\mathbf{1 0}]}{\left\langle 3 p_{1} 0\right]}\right]^{2 S-2} \\
& \times\left\{\left(2 p_{1} p_{0}\right)\langle\mathbf{1} 3\rangle[\mathbf{2 0}](\langle\mathbf{1} 3\rangle[\mathbf{2 0}]+2 S\langle\mathbf{2} 3\rangle[\mathbf{1 0}])\right. \\
& \left.+\left(2 p_{2} p_{0}\right)\langle\mathbf{2} 3\rangle[\mathbf{1 0}](2 S\langle\mathbf{1 3}\rangle[\mathbf{2 0}]+\langle\mathbf{2} 3\rangle[\mathbf{1 0} 0])\right\}+B_{\infty} .
\end{aligned}
$$

This describes the leading effect of the dipole in the limit where the momentum $p_{0}$ is soft. Away from this limit, the expression makes sense for $B_{\infty}=0$ as long as $S \leq 1$, whereas for $S \geq 3 / 2$ one needs to adjust $B_{\infty}$ so as to cancel the unphysical pole at $\left\langle 3 p_{1} 0\right] \rightarrow 0$. A possible boundary term can be constructed using the techniques discussed in appendix B, and is displayed in full glory in eq. (B.11). 


\subsection{Gravitational Compton scattering}

Calculation of gravitational Compton scattering amplitudes $\mathcal{M}\left(\mathbf{1}_{X} \mathbf{2}_{X} 3_{h} 0_{h}\right)$ follows the steps laid out in the previous subsection, with only minor modifications. We again work with the $\{0 \bar{m} \bar{m}\}$ shift of the 012 legs. Consider matter particles minimally coupled to gravity as in eq. (4.1). The leading soft factor $S_{+2}^{(0)}$ in eq. (4.8) is easily calculated as

$$
S_{+2}^{(0)}=-\frac{\left\langle 3 p_{1} 0\right]^{2}[03]^{2}}{M_{\mathrm{Pl}}\left(2 p_{1} p_{0}\right)\left(2 p_{2} p_{0}\right)\left(2 p_{3} p_{0}\right)} .
$$

The absence of subleading soft factors for minimal coupling is just a tad less trivial to demonstrate than in the photon case. Starting from the expression in eq. (4.10) one notes that for our shift $\tilde{\mathcal{D}}_{1}=\tilde{\mathcal{D}}_{2}=0$, while the remaining one reduces to

$$
\tilde{\mathcal{D}}_{3}=[0 \mathbf{1}]\left(\tilde{\lambda}_{0} \frac{\partial}{\partial \chi_{1}}\right)+[0 \mathbf{2}]\left(\tilde{\lambda}_{0} \frac{\partial}{\partial \chi_{2}}\right)+[03]\left(\tilde{\lambda}_{0} \frac{\partial}{\partial \lambda_{3}}\right) .
$$

Thus, $\tilde{\mathcal{D}}_{3}$ is proportional to the total angular momentum operator, and it annihilates 3-point amplitudes. As in the photon case, the IR behavior of gravitational Compton amplitudes is fully determined by the leading soft factor, as long as the graviton is minimally coupled to matter. Given eq. (4.1) and the zero-order shift in eq. (3.9), the relevant hard factors are evaluated as

$$
\begin{aligned}
& \hat{\mathcal{M}}^{0,0}\left(\mathbf{1}_{X} \mathbf{2}_{X} 3_{h}^{-}\right)=-\frac{1}{M_{\mathrm{Pl}}} \frac{\left\langle 3 p_{1} 0\right]^{2}}{[30]^{2}}\left[-\frac{\langle\mathbf{1 3}\rangle[\mathbf{2} 0]+\langle\mathbf{2 3}\rangle[\mathbf{1} 0]}{\left\langle 3 p_{1} 0\right]}\right]^{2 S}, \\
& \hat{\mathcal{M}}^{0,0}\left(\mathbf{1}_{X} \mathbf{2}_{X} 3_{h}^{+}\right)=-\frac{1}{M_{\mathrm{Pl}}} \frac{[30]^{2}}{\left\langle 3 p_{1} 0\right]^{2}} \frac{\langle\mathbf{2 1}\rangle^{2 S}}{m^{2 S-4}} .
\end{aligned}
$$

Thus, the gravitational Compton scattering amplitudes are reconstructed as

$$
\begin{aligned}
\hat{\mathcal{M}}^{0, \epsilon}\left(\mathbf{1}_{X} \mathbf{2}_{X} 3_{h}^{-} 0_{h}^{+}\right) & =\frac{S_{+2}^{(0)}}{\epsilon^{3}} \hat{\mathcal{M}}^{0,0}\left(\mathbf{1}_{X} \mathbf{2}_{X} 3_{h}^{-}\right) \\
& =\frac{(-1)^{2 S}\left\langle 3 p_{1} 0\right]^{4}}{\epsilon^{3} M_{\mathrm{Pl}}^{2}\left(2 p_{1} p_{0}\right)\left(2 p_{2} p_{0}\right)\left(2 p_{3} p_{0}\right)}\left[\frac{\langle\mathbf{1 3}\rangle[\mathbf{2} 0]+\langle\mathbf{2} 3\rangle[\mathbf{1 0}]}{\left\langle 3 p_{1} 0\right]}\right]^{2 S}+B_{\infty} \\
\hat{\mathcal{M}}^{0, \epsilon}\left(\mathbf{1}_{X} \mathbf{2}_{X} 3_{h}^{+} 0_{h}^{+}\right) & =\frac{S_{+2}^{(0)}}{\epsilon^{3}} \hat{\mathcal{M}}^{0,0}\left(\mathbf{1}_{X} \mathbf{2}_{X} 3_{h}^{+}\right) \\
& =\frac{[30]^{4}}{\epsilon^{3} M_{\mathrm{Pl}}^{2}\left(2 p_{1} p_{0}\right)\left(2 p_{2} p_{0}\right)\left(2 p_{3} p_{0}\right)} \frac{\langle\mathbf{2 1}\rangle^{2 S}}{m^{2 S-4}}+B_{\infty}^{\prime} .
\end{aligned}
$$

The IR part agrees with the expression derived in [22] by matching the residues at the kinematic poles. The amplitude away from the soft limit is obtained using $\mathcal{M}=\hat{\mathcal{M}}^{0,1}$. Much as for photons, it is consistent to set $B_{\infty}^{\prime}=0$ for any spin, however for $S>2$ the boundary term $B_{\infty}$ has to be non-zero so as to cancel the unphysical $\left\langle 3 p_{1} 0\right]$ pole, see appendix B.2 for an explicit expression.

Let us also work out a simple example beyond the leading soft factor. Consider a massive particle with spin $S \geq 1$ and non-minimal quadrupole interactions with the graviton, 
corresponding to $Q \neq 0$ in eq. (4.21). In this case, the plus-helicity sub-subleading soft factor in eq. (4.22) for gravitational Compton scattering reduces to

$$
S_{+2}^{(2)}=\frac{Q}{4 M_{\mathrm{Pl}} m^{2}}\left\{\frac{[\mathbf{1 0}]^{2}\left(\tilde{\chi}_{l L_{1}} \tilde{\lambda}_{0}\right)\left(\tilde{\chi}_{l L_{2}} \tilde{\lambda}_{0}\right)}{p_{0} p_{1}}+\frac{[\mathbf{2 0}]^{2}\left(\tilde{\chi}_{2 K_{1}} \tilde{\lambda}_{0}\right)\left(\tilde{\chi}_{2 K_{2}} \tilde{\lambda}_{0}\right)}{p_{0} p_{2}}\right\}
$$

where we displayed the little group indices acting non-trivially on the hard factor. The latter for plus helicity photons is generalized as

$$
\hat{\mathcal{M}}^{0,0}\left(\mathbf{1}_{X} \mathbf{2}_{X} 3_{h}^{+}\right)=-\frac{1}{M_{\mathrm{Pl}} m^{2 S-4}}\left\{\frac{[30]^{2}}{\left\langle 3 p_{1} 0\right]^{2}}\langle\mathbf{2 1}\rangle^{2 S}+\frac{Q}{2} \frac{[30]^{4}}{\left\langle 3 p_{1} 0\right]^{4}}\langle\mathbf{1} 3\rangle^{2}\langle\mathbf{2} 3\rangle^{2}\langle\mathbf{2 1}\rangle^{2 S-2}\right\} .
$$

Applying this to derive, for example, the same-helicity Compton amplitude we find

$$
\begin{aligned}
\hat{\mathcal{M}}^{0, \epsilon}\left(\mathbf{1}_{X} \mathbf{2}_{X} 3_{h}^{+} 0_{h}^{+}\right)= & {\left[\frac{S_{+2}^{(0)}}{\epsilon^{3}}+\frac{S_{+2}^{(2)}}{\epsilon}\right] \hat{\mathcal{M}}^{0,0}\left(\mathbf{1}_{X} \mathbf{2}_{X} 3_{h}^{+}\right)=\frac{[30]^{4}}{\epsilon^{3} M_{\mathrm{Pl}}^{2}\left(2 p_{1} p_{0}\right)\left(2 p_{2} p_{0}\right)\left(2 p_{3} p_{0}\right)} \frac{\langle\mathbf{2 1}\rangle^{2 S}}{m^{2 S-4}} } \\
& +\frac{Q}{2} \frac{[30]^{6}}{\epsilon^{3} M_{\mathrm{Pl}}^{2}\left(2 p_{1} p_{0}\right)\left(2 p_{2} p_{0}\right)\left(2 p_{3} p_{0}\right)} \frac{\langle\mathbf{1 3}\rangle^{2}\langle\mathbf{2} 3\rangle^{2}}{\left\langle 3 p_{1} 0\right]^{2}} \frac{\langle\mathbf{2 1}\rangle^{2 S-2}}{m^{2 S-4}} \\
& -\frac{Q}{2} \frac{[30]^{2}}{\epsilon M_{\mathrm{Pl}}^{2}\left\langle 3 p_{1} 0\right]^{2}}\left[\frac{[\mathbf{1 0}]^{2}\left\langle\mathbf{2} p_{1} 0\right]^{2}}{2 p_{0} p_{1}}+\frac{[\mathbf{2 0} 0]^{2}\left\langle\mathbf{1} p_{2} 0\right]^{2}}{2 p_{0} p_{2}}\right] \frac{\langle\mathbf{2 1}\rangle^{2 S-2}}{m^{2 S-2}} \\
& +\mathcal{O}\left(Q^{2}\right)+B_{\infty}^{\prime} .
\end{aligned}
$$

Unlike in the minimal case, in the presence of the quadrupole the unphysical $\left\langle 3 p_{1} 0\right]$ pole appears also in the same-helicity amplitude, and it is present already for $S=1$. If one uses this formula away from the soft limit, one needs to include an appropriate boundary term $B_{\infty}^{\prime}$ to cancel this pole for any $S \geq 1$

\subsection{Higher-point}

To conclude this section, we discuss a couple of examples of 5-point amplitude calculations using the soft theorems of section 4. The goal is to provide an illustration how these formulas should be employed in the case of higher-point amplitudes. The examples involve a matter particle $X$ with mass $m$, arbitrary spin $S$, and the minimal coupling to photons and gravitons.

Our first example is the amplitude describing the production of three photons in the $X \bar{X}$ annihilation: $\mathcal{M}\left(\mathbf{1}_{X} \mathbf{2}_{\bar{X}} 3_{\gamma} 4_{\gamma} 0_{\gamma}\right)$. We use the $\{0 \bar{m} \bar{m}\}(\{\overline{0} m m\})$ shift on the 012 legs to calculate emission of a soft photon $0_{\gamma}$ with plus (minus) helcity. The leading soft factors for emission of $0_{\gamma}$ are given by

$$
S_{+1}^{(0)}=\frac{\sqrt{2} e\left[0 p_{1} p_{2} 0\right]}{\left(2 p_{1} p_{0}\right)\left(2 p_{2} p_{0}\right)}, \quad S_{-1}^{(0)}=\frac{\sqrt{2} e\left\langle 0 p_{1} p_{2} 0\right\rangle}{\left(2 p_{1} p_{0}\right)\left(2 p_{2} p_{0}\right)} .
$$

These expressions are in fact true for $\mathcal{M}\left(\mathbf{1}_{X} \mathbf{2}_{\bar{X}} 3_{\gamma} \ldots n_{\gamma} 0_{\gamma}\right)$ with any $n-2 \geq 1$ number of hard photons. The subleading factors are moot for the minimal coupling. That is because 
the only charged particles are at the positions 1 and 2 , however $\mathcal{D}_{1,2}=0$ for the shift we have chosen. Given eq. (5.4), the hard factor in the soft recursion is given by

$$
\hat{\mathcal{M}}^{0,0}\left(\mathbf{1}_{X} \mathbf{2}_{\bar{X}} 3_{\gamma}^{+} 4_{\gamma}^{+}\right)=\frac{2 e^{2}[34]^{2}}{\left(2 \tilde{p}_{1} p_{3}\right)\left(2 \tilde{p}_{1} p_{4}\right)} \frac{\langle\mathbf{2 1}\rangle^{2 S}}{m^{2 S-2}},
$$

where

$$
\left.2 \tilde{p}_{1} p_{l} \equiv 2 \tilde{p}_{1} p_{l}\right|_{z=0, \epsilon=0}=2 p_{1} p_{l}+2 p_{2} p_{0} \frac{\left[0 p_{1} p_{l} 0\right]}{\left[0 p_{1} p_{2} 0\right]} .
$$

Putting it all together,

$$
\begin{aligned}
\hat{\mathcal{M}}^{0, \epsilon}\left(\mathbf{1}_{X} \mathbf{2}_{\bar{X}} 3_{\gamma}^{+} 4_{\gamma}^{+} 0_{\gamma}^{+}\right) & =\frac{2 \sqrt{2} e^{3}[34]^{2}\left[0 p_{1} p_{2} 0\right]}{\epsilon^{2}\left(2 p_{1} p_{0}\right)\left(2 p_{2} p_{0}\right)\left(2 \tilde{p}_{1} p_{3}\right)\left(2 \tilde{p}_{1} p_{4}\right)} \frac{\langle\mathbf{2 1}\rangle^{2 S}}{m^{2 S-2}}+\mathcal{O}\left(\epsilon^{0}\right), \\
\hat{\mathcal{M}}^{0, \epsilon}\left(\mathbf{1}_{X} \mathbf{2}_{\bar{X}} 3_{\gamma}^{+} 4_{\gamma}^{+} 0_{\gamma}^{-}\right) & =\frac{2 \sqrt{2} e^{3}[34]^{2}\left\langle 0 p_{1} p_{2} 0\right\rangle}{\epsilon^{2}\left(2 p_{1} p_{0}\right)\left(2 p_{2} p_{0}\right)\left(2 \tilde{p}_{1} p_{3}\right)\left(2 \tilde{p}_{1} p_{4}\right)} \frac{\langle\mathbf{2 1}\rangle^{2 S}}{m^{2 S-2}}+\mathcal{O}\left(\epsilon^{0}\right) .
\end{aligned}
$$

The similar approach can be used when photons are replaced with gravitons: $\mathcal{M}\left(\mathbf{1}_{X} \mathbf{2}_{X} 3_{h} 4_{h} 0_{h}\right)$. Below we sketch the derivation, however the final formulas are too lengthy to quote here. The leading soft factor for emission of a plus helicity graviton is

$$
S_{+2}^{(0)}=\frac{1}{M_{\mathrm{Pl}}\langle 03\rangle^{2}}\left\{\frac{\left\langle 3 p_{1} 0\right]^{2}}{2 p_{0} p_{1}}+\frac{\left\langle 3 p_{2} 0\right]^{2}}{2 p_{0} p_{2}}+\frac{\langle 34\rangle^{2}[40]^{2}}{2 p_{0} p_{4}}\right\} .
$$

Here we set $y=\lambda_{3}$ in eq. (4.8), since the expression is true for an arbitrary spinor $y$. A major difference compared to the photon calculation before is that now the subleading soft factors are non-trivial. Indeed, picking again $y=\lambda_{3}$ in eq. (4.10), the subleading soft factor can be written as

$$
S_{+2}^{(1)}=\frac{\langle 34\rangle[40]}{M_{\mathrm{Pl}}\left(2 p_{0} p_{4}\right)\langle 03\rangle} \tilde{\mathcal{D}}_{4}, \quad \tilde{\mathcal{D}}_{4}=[04]\left[0 \partial_{4}\right]+\frac{\left\langle 4 p_{2} 0\right][40]}{\left[0 p_{1} p_{2} 0\right]}[0 \mathbf{1}]\left[0 \partial_{\mathbf{1}}\right]-\frac{\left\langle 4 p_{1} 0\right][40]}{\left[0 p_{1} p_{2} 0\right]}[0 \mathbf{2}]\left[0 \partial_{\mathbf{2}}\right] .
$$

The soft expansion of the 5-point amplitude $\mathcal{M}^{0, \epsilon}\left(\mathbf{1}_{X} \mathbf{2}_{X} 3_{h}^{+} 4_{h}^{+} 0_{h}^{+}\right)$is calculated using eq. (4.7) with the soft factors above acting on $\mathcal{M}^{0,0}\left(\mathbf{1}_{X} \mathbf{2}_{X} 3_{h}^{+} 4_{h}^{+}\right)$, which in turn can be obtained from eq. (5.13). One can explicitly verify that $\tilde{\mathcal{D}}_{4} \mathcal{M}^{0,0}\left(\mathbf{1}_{X} \mathbf{2}_{X} 3_{h}^{+} 4_{h}^{+}\right) \neq 0$, thus the subleading soft factor is not moot in this case.

\section{Exponential representation}

It has been known for a long time that the multipole expansion of classical spinning bodies, such as a Kerr black hole, can be resumed in a simple exponential form [45]. However, the corresponding description in terms of on-shell amplitudes was obtained just recently in [24]. That reference noticed that the on-shell 3-point amplitude describing minimal graviton interactions with matter of any spin can be recast in a compact exponential form, with the angular momentum operator in the exponent. Subsequently, they demonstrated that also the 4-point gravitational Compton scattering amplitude can be recast in an exponential form. This formal rephrasing turns out to be useful to compute the scattering angle of two spinning black holes [24]. As we are going to show, our soft recursion leads to a simple proof 
of the exponentiation of the Compton amplitudes for arbitrary spin of the matter particle. In the following, we demonstrate it for the photon amplitudes, however the derivation for gravity is totally analogous. We also discuss the exponential representation of the soft theorems for any (integer) helicity of the soft particle.

\subsection{Photon exponentiation}

Let us first rewrite the photon's minimal coupling as

$$
\mathcal{M}\left(\mathbf{1}_{X} \mathbf{2}_{\bar{X}} 3_{\gamma}^{-}\right)=\frac{2 e q\left(p_{1} \epsilon_{3}^{-}\right)}{m^{2 S}}[\mathbf{2 1}]^{2 S}, \quad \mathcal{M}\left(\mathbf{1}_{X} \mathbf{2}_{\bar{X}} 3_{\gamma}^{+}\right)=\frac{2 e q\left(p_{1} \epsilon_{3}^{+}\right)}{m^{2 S}}\langle\mathbf{2 1}\rangle^{2 S} .
$$

This is obtained from the leading term in eq. (4.23) by replacing the $\zeta$ terms with the polarization vectors defined in eq. (2.5). ${ }^{11}$ Eq. (6.1) can be equivalently recast as

$$
\begin{aligned}
& \mathcal{M}\left(\mathbf{1}_{X} \mathbf{2}_{\bar{X}} 3_{\gamma}^{-}\right)=\frac{2 e q\left(p_{1} \epsilon_{3}^{-}\right)}{m^{2 S}} \exp \left(-i \frac{\epsilon_{3}^{-\mu} p_{3}^{\nu} J_{1}^{\mu \nu}}{\left(p_{1} \epsilon_{3}^{-}\right)}\right)\langle\mathbf{2 1}\rangle^{2 S}, \\
& \mathcal{M}\left(\mathbf{1}_{X} \mathbf{2}_{\bar{X}} 3_{\gamma}^{+}\right)=\frac{2 e q\left(p_{1} \epsilon_{3}^{+}\right)}{m^{2 S}} \exp \left(-i \frac{\epsilon_{3}^{+\mu} p_{3}^{\nu} J_{1}^{\mu \nu}}{\left(p_{1} \epsilon_{3}^{+}\right)}\right)[\mathbf{2 1}]^{2 S},
\end{aligned}
$$

where $J_{i}^{\mu \nu}$ are the angular momentum operators introduced in eq. (4.12). Note that we could equivalently replace $1 \rightarrow 2$ in the exponent. The proof relies on the identities $\langle\mathbf{1 2}\rangle-[\mathbf{1 2}]=\frac{\langle 31 \zeta]}{m^{2}[3 \zeta]}[3 \mathbf{1}][32]=\frac{\langle\zeta 13]}{m^{2}\langle 3 \zeta\rangle}\langle 3 \mathbf{1}\rangle\langle 32\rangle$ and

$$
\epsilon_{3}^{-\mu} p_{3}^{\nu} J_{\chi_{l}}^{\mu \nu}=\frac{i}{\sqrt{2}}\langle 3 \mathbf{l}\rangle\left(\lambda_{3} \frac{\partial}{\partial \chi_{l}}\right), \quad \epsilon_{3}^{+\mu} p_{3}^{\nu} J_{\tilde{\chi}_{l}}^{\mu \nu}=\frac{i}{\sqrt{2}}[3 \mathbf{l}]\left(\tilde{\lambda}_{3} \frac{\partial}{\partial \tilde{\chi}_{l}}\right), \quad \epsilon_{3}^{-\mu} p_{3}^{\nu} J_{\tilde{\chi}_{l}}^{\mu \nu}=\epsilon_{3}^{+\mu} p_{3}^{\nu} J_{\chi_{l}}^{\mu \nu}=0
$$

from which it follows $\chi_{2}\left(1-i \frac{\epsilon_{3}^{-\mu} p_{3}^{\nu} J_{1}^{\mu \nu}}{\left(p_{1} \epsilon_{3}^{-}\right)}\right) \chi_{1}=[\mathbf{2 1}], \tilde{\chi}_{2}\left(1-i \frac{\epsilon_{3}^{+\mu} p_{3}^{\nu} J_{1}^{\mu \nu}}{\left(p_{1} \epsilon_{3}^{+}\right)}\right) \tilde{\chi}_{1}=\langle\mathbf{2 1}\rangle$.

We move to the calculation of the opposite helicity Compton amplitude using the soft recursion in eq. (3.7) with the $\{0 \bar{m} \bar{m}\}$ shift on the 012 legs. For the soft 3-point amplitude we use the exponentiated form in eq. (6.2), while for the hard factor we use the minimal form in eq. (6.1). Then the recursion reduces to

$$
\begin{aligned}
\hat{\mathcal{M}}^{0, \epsilon}\left(\mathbf{1}_{X} \mathbf{2}_{\bar{X}} 3_{\gamma}^{-} 0_{\gamma}^{+}\right)=\frac{(-1)^{2 S} 4 q^{2} e^{2}}{\epsilon m^{2 S}}\{ & \left.\frac{\left(\hat{p}_{2} \epsilon_{3}^{-}\right)\left(\hat{p}_{1} \hat{\epsilon}_{0}^{+}\right)}{u-m^{2}} \exp \left(-i \frac{\epsilon_{3}^{-\mu} p_{3}^{\nu} J_{2}^{\mu \nu}}{\left(\hat{p}_{2} \epsilon_{3}^{-}\right)}\right)\langle\mathbf{2 1}\rangle^{2 S}\right|_{z=z_{1}} \\
& \left.+\left.\frac{\left(\hat{p}_{1} \epsilon_{3}^{-}\right)\left(\hat{p}_{2} \hat{\epsilon}_{0}^{+}\right)}{t-m^{2}} \exp \left(-i \frac{\epsilon_{3}^{-\mu} p_{3}^{\nu} J_{1}^{\mu \nu}}{\left(\hat{p}_{1} \epsilon_{3}^{-}\right)}\right)\langle\mathbf{2 1}\rangle^{2 S}\right|_{z=z_{2}}\right\}+B_{\infty},
\end{aligned}
$$

where we already contracted the $\chi_{0 l}$ spinors using eq. (2.7). The crucial identities for this calculation are

$$
\begin{array}{ll}
\left.\left(\hat{p}_{1} \hat{\epsilon}_{0}^{+}\right)\right|_{z=z_{1}}=\frac{\left\langle y p_{1} 0\right]}{\epsilon \sqrt{2}\langle 0 y\rangle}=\left.\frac{1}{\epsilon}\left(p_{1} \epsilon_{0}^{+}\right)\right|_{\zeta=y},\left.\quad\left(\hat{p}_{2} \hat{\epsilon}_{0}^{+}\right)\right|_{z=z_{2}}=\frac{\left\langle y p_{2} 0\right]}{\epsilon \sqrt{2}\langle 0 y\rangle}=\left.\frac{1}{\epsilon}\left(p_{2} \epsilon_{0}^{+}\right)\right|_{\zeta=y}, \\
\left.\left(\hat{p}_{2} \epsilon_{3}^{-}\right)\right|_{z=z_{1}}=\frac{\left\langle 3 p_{2} 0\right]}{\sqrt{2}[30]}=\left.\left(p_{2} \epsilon_{3}^{-}\right)\right|_{\tilde{\zeta}=\tilde{\lambda}_{0}},\left.\quad\left(\hat{p}_{1} \epsilon_{3}^{-}\right)\right|_{z=z_{2}}=\frac{\left\langle 3 p_{1} 0\right]}{\sqrt{2}[30]}=\left.\left(p_{1} \epsilon_{3}^{-}\right)\right|_{\tilde{\zeta}=\tilde{\lambda}_{0}} .
\end{array}
$$

\footnotetext{
${ }^{11}$ While polarization vectors themselves depends on the gauge (or the reference spinor $\zeta$ ), the 3-point function is gauge-independent because $p_{1} p_{3}=0$ on the 3-body kinematics.
} 
One should stress that these are valid for any reference spinors $\zeta$ and $\tilde{\zeta}$; no "gauge-fixing" was performed along the way. Then eq. (6.4) can be written as

$$
\begin{aligned}
\hat{\mathcal{M}}^{0, \epsilon}\left(\mathbf{1}_{X} \mathbf{2}_{\bar{X}} 3_{\gamma}^{-} 0_{\gamma}^{+}\right)=\frac{(-1)^{2 S} 2 q^{2} e^{2}\left\langle 3 p_{1} 0\right]}{\epsilon^{2} m^{2 S}[30]\langle 0 y\rangle}\{ & -\frac{\left\langle y p_{1} 0\right]}{u-m^{2}} \exp \left(-i \frac{\epsilon_{3}^{-\mu} p_{3}^{\nu} J_{2}^{\mu \nu}}{\left.\left(p_{2} \epsilon_{3}^{-}\right)\right|_{\tilde{\zeta}=\tilde{\lambda}_{0}}}\right)\langle\mathbf{2 1}\rangle^{2 S} \\
& \left.+\frac{\left\langle y p_{2} 0\right]}{t-m^{2}} \exp \left(-i \frac{\epsilon_{3}^{-\mu} p_{3}^{\nu} J_{1}^{\mu \nu}}{\left.\left(p_{1} \epsilon_{3}^{-}\right)\right|_{\tilde{\zeta}=\tilde{\lambda}_{0}}}\right)\langle\mathbf{2 1}\rangle^{2 S}\right\}+B_{\infty} .
\end{aligned}
$$

In the exponentials, the numerators do not depend on the reference spinors thanks to the antisymmetry of $J^{\mu \nu}$. On the other hand, in the denominators, $\left(p_{i} \epsilon_{3}^{-}\right)$do depend on the reference spinors. Our calculation unambiguously dictates that these have to be evaluated at a particular reference spinor, $\tilde{\zeta}=\tilde{\lambda}_{0} \cdot{ }^{12}$ The two exponentials are in fact equal, so we can pull them in front of the curly bracket. Then, summing the $t$ - and $u$-poles, the dependence on the shift spinor $y$ cancels out and we obtain the formula for the Compton amplitude in the exponential form [26]:

$$
\hat{\mathcal{M}}^{0, \epsilon}\left(\mathbf{1}_{X} \mathbf{2}_{\bar{X}} 3_{\gamma}^{-} 0_{\gamma}^{+}\right)=(-1)^{2 S} \frac{2 q^{2} e^{2}\left\langle 3 p_{1} 0\right]^{2}}{\epsilon^{2} m^{2 S}\left(t-m^{2}\right)\left(u-m^{2}\right)} \exp \left(-i \frac{\epsilon_{3}^{-\mu} p_{3}^{\nu} J_{1}^{\mu \nu}}{\left.\left(p_{1} \epsilon_{3}^{-}\right)\right|_{\tilde{\zeta}=\tilde{\lambda}_{0}}}\right)\langle\mathbf{2 1}\rangle^{2 S}+B_{\infty} .
$$

This proves exponentiation of IR terms in Compton scattering for any matter spin. Recall that $B_{\infty}$ starts at $\mathcal{O}\left(\epsilon^{1}\right)$, and thus qualifies as a UV term. Given its explicit form in eq. (5.5), it does not appear to have a simple exponential representation. Finally, notice that the exponential can be rewritten in a more explicit form as

$$
\hat{\mathcal{M}}^{0, \epsilon}\left(\mathbf{1}_{X} \mathbf{2}_{\bar{X}} 3_{\gamma}^{-} 0_{\gamma}^{+}\right)=(-1)^{2 S} \frac{2 q^{2} e^{2}\left\langle 3 p_{1} 0\right]^{2}}{\epsilon^{2} m^{2 S}\left(t-m^{2}\right)\left(u-m^{2}\right)} \exp \left(\frac{[30]\langle 3 \mathbf{1}\rangle\left(\lambda_{3} \partial_{\chi_{1}}\right)}{\left\langle 3 p_{1} 0\right]}\right)\langle\mathbf{2 1}\rangle^{2 S}+B_{\infty} .
$$

\subsection{Soft exponentiation}

Ref. [14] showed that the soft theorem for gravitons and gluons could be written in terms of an exponential operator acting on lower point amplitudes. However, the proof is only valid for the minimal-helicity-violating (MHV) sector, as it relies on the vanishing of the boundary terms discussed in [11]. Using the soft recursion we can show that the exponential form is actually valid beyond the MHV sector. In fact, the exponential operator can be extended for amplitudes with massive particles of any spin and with a soft particle of any integer helicity $|h| \leq 2$. We begin by rewriting the minimal 3-point amplitude for $h \geq 0$ as

$$
\mathcal{M}\left(\mathbf{1}_{X} \mathbf{2}_{\bar{X}} 3^{-h}\right)=g_{X}\left(\frac{\left\langle 3 p_{1} \zeta\right]}{\langle 3 \zeta\rangle}\right)^{h} \frac{[\mathbf{2 1}]^{2 S}}{m^{2 S-1+h}}, \quad \mathcal{M}\left(\mathbf{1}_{X} \mathbf{2}_{\bar{X}} 3^{h}\right)=g_{X}\left(\frac{\left\langle\zeta p_{1} 3\right]}{[3 \zeta]}\right)^{h} \frac{\langle\mathbf{2 1}\rangle^{2 S}}{m^{2 S-1+h}}
$$

where $g_{X}=\sqrt{2} e q$ for $|h|=1$, and $g_{X}=m / M_{\mathrm{Pl}}$ for $|h|=2$. The soft expansion in eq. (4.4) and eq. (4.24) can be collectively written as:

$$
\hat{\mathcal{M}}^{0, \epsilon}\left(1 \ldots n 0^{+}\right)=\frac{1}{\epsilon^{1+h} m^{1+h}} \sum_{l=1}^{n} \frac{g_{l}\left\langle y p_{l} 0\right]^{h}}{\left(2 p_{0} p_{l}\right)\langle 0 y\rangle^{h}} \hat{\mathcal{M}}^{z_{l}, \epsilon}\left(1 \ldots P_{0 l} \ldots n\right)+\mathcal{O}\left(\epsilon^{0}\right) .
$$

\footnotetext{
${ }^{12}$ This iluminates one tiny detail in the derivation of ref. [24], where the choice of the reference spinor was made ad-hoc.
} 
Now, the Taylor expansion in eq. (4.5) can be (trivially) recast in the exponential form:

$$
\hat{\mathcal{M}}^{z_{l}, \epsilon}\left(1 \ldots P_{0 l} \ldots n\right)=\exp \left(\epsilon \frac{\langle 0 y\rangle}{\left\langle y p_{l} 0\right]} \tilde{\mathcal{D}}_{l}\right) \hat{\mathcal{M}}^{0,0}(1 \ldots n)+\mathcal{O}\left(\epsilon^{3}\right)
$$

This way, for minimal coupling, we obtain a similar form of the soft theorem as the one in ref. [14]:

$$
\mathcal{M}^{0, \epsilon}\left(1 \ldots n 0^{+}\right)=\frac{1}{\epsilon^{1+h} m^{1+h}} \sum_{l=1}^{n} \frac{g_{X}\left\langle y p_{l} 0\right]^{h}}{\left(2 p_{0} p_{l}\right)\langle 0 y\rangle^{h}} \exp \left(\epsilon \frac{\langle 0 y\rangle}{\left\langle y p_{l} 0\right]} \tilde{\mathcal{D}}_{l}\right) \hat{\mathcal{M}}^{0,0}(1 \ldots n)+\mathcal{O}\left(\epsilon^{0}\right)
$$

where the differential operator $\tilde{\mathcal{D}}_{l}$ is given in eq. (4.6). This form may be useful to study amplitudes in the Mellin space along the lines of ref. [46]. Moreover, our results in eq. (4.28) for the electromagnetic dipole suggest that a generalization (for soft photons) consists in replacing

$$
\tilde{\mathcal{D}}_{l} \rightarrow \tilde{\mathcal{D}}_{l}+\frac{S a_{l}}{q_{l} m}[0 \mathbf{l}]^{2},
$$

where for massive $l$ the suppressed little group indices can be read off from eq. (4.28). Similarly, for gravitons, eq. (4.22) can be reproduced via $\tilde{\mathcal{D}}_{l} \rightarrow \tilde{\mathcal{D}}_{l}+\epsilon Q_{l} \frac{\left\langle y p_{l} 0\right]}{2\langle 0 y\rangle}[0 \mathbf{l}]^{4}$.

\section{Conclusions}

This paper provides a new toolbox to study theories with particles of arbitrary mass and spin. We introduced a class of complex momentum shifts combining a BCFW-like spinor shift with a soft limit of a massless particle present in the process. The important technical novelty is that our shifts are compatible with the massive spinors introduced by ArkaniHamed, Huang, and Huang in ref. [22]. Via the usual road of the Cauchy theorem, these shifts allow one to derive novel recursion relations for scattering amplitudes, even when all but one particles involved are massive.

This formal development will find multiple practical applications, we hope. In this paper we applied it to derive a new incarnation of the soft theorems for emission of a soft photon or a graviton. They function, out of the box, with amplitudes expressed in the on-shell spinor formalism, also with massive spinors, thus generalizing the massless results of ref. [16]. This allows us to systematically explore soft theorems for general theories, and make a direct connection with the multipole expansion of the on-shell 3-point interaction between matter and photons/gravitons. In particular, our soft theorems lead to an elegant proof that gravitational dipole interactions are incompatible with the assumption of locality, unitarity and Poincaré invariance. We also provide a concise expression for the modification of the soft theorems due to the quadrupole (for gravitons) and dipole (for photons) non-minimal interactions with matter.

Generally, soft theorems only capture the leading terms in the limit where the massless particle momentum goes to zero, because they take into account only the soft particle emission from external legs. However, for Compton scattering, emission from external legs 
accounts for all factorization channels. Therefore, Compton amplitudes derived via soft theorems reflect the complete structure of physical poles required by unitarity. A subtlety appears for spin $S \geq 3 / 2$ (for photons) and $S \geq 5 / 2$ (for gravitons) when these amplitudes develop a spurious pole, which has to be subtracted by adding a judiciously designed UV term. Our approach leads to a new perspective on these UV terms: they arise as boundary terms in our recursion relations. We laid out an algorithm for constructing the UV term for Compton scattering of photons, which leads to a more compact and transparent formula than the one in ref. [34]. We also derived the UV term for Compton scattering via the electromagnetic dipole. These results may be useful for practical applications, for example in nuclear or atomic physics. Furthermore, our soft recursion leads by the shortest route to recasting the Compton amplitude into a form where the increasing matter spin corresponds to an action of an exponentiated angular momentum operator $[24,26]$. We were also able to rewrite more general soft recursions in the exponentiated form, extending the results of ref. [14].

Our soft recursions may help with calculation of multi-leg amplitudes - the topic we only fleetingly touched in this paper. That could in turn boost the amplitude methods of classical calculations of electromagnetic and gravitational radiation along the lines of ref. [23]. These recursions should also be useful to derive more general soft theorems for Goldstone fermions [47] and gravitinos [48]. Furthermore, it would be interesting to generalize our recursions to multiple soft particles [49] (as recently explored with the CHY formalism [50]). More speculatively, the tools we provide may open up new areas of exploration of massive amplitudes. One direction to mention in this context is the link with the asymptotic symmetries $[5,51]$, another is the double copy structure of the soft theorems for massive particles [52].

\section{Acknowledgments}

AF is partially supported by the French Agence Nationale de la Recherche (ANR) under grant ANR-19-CE31-0012 (project MORA). The work of C.S.M. is supported by the Alexander von Humboldt Foundation, in the framework of the Sofja Kovalevskaja Award 2016, endowed by the German Federal Ministry of Education and Research and also supported by the Cluster of Excellence "Precision Physics, Fundamental Interactions, and Structure of Matter" (PRISMA+ EXC 2118/1) funded by the German Research Foundation (DFG) within the German Excellence Strategy (Project ID 39083149).

\section{A Summary of spinor shifts}

In this appendix we list distinct spinor shifts realizing the momentum shift in eq. (3.1). We label them as $\{0 \bar{A} \bar{B}\}$ or $\{\overline{0} A B\}$, where $A(B)=0$ when the $j$-th $(k$-th) particle is massless, and $A(B)=m$ when the $j$-th $(k$-th) particle is massive. The label $\{0 \bar{A} \bar{B}\}$ $(\{\overline{0} A B\})$ corresponds to shifting the holomorphic(antiholomorphic) spinor of the massless particle 0 , and the antiholomorphic(holomorphic) spinors of the $j$-th and $k$-th particles. 
$\{0 \overline{0} \overline{0}\}$.

$$
\begin{aligned}
q_{0} \sigma & =y \tilde{\lambda}_{0}, \quad q_{j} \sigma=\lambda_{j} \tilde{\lambda}_{0}, \quad q_{k} \sigma=\lambda_{k} \tilde{\lambda}_{0} . \\
\lambda_{0}^{z} & =\epsilon \lambda_{0}-z y, \\
\tilde{\lambda}_{j}^{z} & =\tilde{\lambda}_{j}-\frac{(\epsilon-1)\langle 0 k\rangle-z\langle y k\rangle}{\langle j k\rangle} \tilde{\lambda}_{0}, \\
\tilde{\lambda}_{k}^{z} & =\tilde{\lambda}_{k}+\frac{(\epsilon-1)\langle 0 j\rangle-z\langle y j\rangle}{\langle j k\rangle} \tilde{\lambda}_{0} .
\end{aligned}
$$

$\{\overline{0} 00\}$.

$$
\begin{aligned}
q_{0} \sigma & =\lambda_{0} \tilde{y}, \quad q_{j} \sigma=\lambda_{0} \tilde{\lambda}_{j}, \quad q_{k} \sigma=\lambda_{0} \tilde{\lambda}_{k} . \\
\tilde{\lambda}_{0}^{z} & =\epsilon \tilde{\lambda}_{0}-z \tilde{y} \\
\lambda_{j}^{z} & =\lambda_{j}-\frac{(\epsilon-1)[0 k]-z[y k]}{[j k]} \lambda_{0}, \\
\lambda_{k}^{z} & =\lambda_{k}+\frac{(\epsilon-1)[0 j]-z[y j]}{[j k]} \lambda_{0} .
\end{aligned}
$$

$\{0 \bar{m} \overline{0}\}$

$$
\begin{aligned}
q_{0} \sigma & =y \tilde{\lambda}_{0}, \quad q_{j} \sigma=p_{j} \sigma \tilde{\lambda}_{0} \tilde{\lambda}_{0}, \quad q_{k} \sigma=\lambda_{k} \tilde{\lambda}_{0} . \\
\lambda_{0}^{z} & =\epsilon \lambda_{0}-z y, \\
\tilde{\chi}_{j}^{z} & =\tilde{\chi}_{j}-\frac{(\epsilon-1)\langle k 0\rangle-z\langle k y\rangle}{\left\langle k p_{j} 0\right]}[\mathbf{j} \tilde{0}] \tilde{\lambda}_{0} \\
\tilde{\lambda}_{k}^{z} & =\tilde{\lambda}_{k}-\frac{(\epsilon-1)\left\langle 0 p_{j} 0\right]-z\left\langle y p_{j} 0\right]}{\left\langle k p_{j} 0\right]} \tilde{\lambda}_{0} .
\end{aligned}
$$

$\{\overline{0} m 0\}$.

$$
\begin{aligned}
q_{0} \sigma & =\lambda_{0} \tilde{y}, \quad q_{j} \sigma=\lambda_{0} \lambda_{0} p_{j} \bar{\sigma}, \quad q_{k} \sigma=\lambda_{0} \tilde{\lambda}_{k} . \\
\tilde{\lambda}_{0}^{z} & =\epsilon \tilde{\lambda}_{0}-z \tilde{y} \\
\chi_{j}^{z} & =\chi_{j}-\frac{(\epsilon-1)[k 0]-z[k y]}{\left\langle 0 p_{j} k\right]}[\mathbf{j} 0] \lambda_{0}, \\
\lambda_{k}^{z} & =\lambda_{k}-\frac{(\epsilon-1)\left\langle 0 p_{j} 0\right]-z\left\langle 0 p_{j} y\right]}{\left\langle 0 p_{j} k\right]} \lambda_{0} .
\end{aligned}
$$

$\{0 \bar{m} \bar{m}\}$.

$$
\begin{aligned}
q_{0} \sigma & =y \tilde{\lambda}_{0}, \quad q_{j} \sigma=p_{j} \sigma \tilde{\lambda}_{0} \tilde{\lambda}_{0}, \quad q_{k} \sigma=p_{k} \sigma \tilde{\lambda}_{0} \tilde{\lambda}_{0} . \\
\lambda_{0}^{z} & =\epsilon \lambda_{0}-z y, \\
\tilde{\chi}_{j}^{z} & =\tilde{\chi}_{j}+\frac{(\epsilon-1)\left\langle 0 p_{k} 0\right]-z\left\langle y p_{k} 0\right]}{\left[0 p_{j} p_{k} 0\right]}[\mathbf{j} 0] \tilde{\lambda}_{0}, \\
\tilde{\chi}_{k}^{z} & =\tilde{\chi}_{k}-\frac{(\epsilon-1)\left\langle 0 p_{j} 0\right]-z\left\langle y p_{j} 0\right]}{\left[0 p_{j} p_{k} 0\right]}[\mathbf{k} 0] \tilde{\lambda}_{0} .
\end{aligned}
$$


$\{\overline{\mathbf{0}} \boldsymbol{m} \boldsymbol{m}\}$.

$$
\begin{aligned}
q_{0} \sigma & =\lambda_{0} \tilde{y}, \quad q_{j} \sigma=\lambda_{0} p_{j} \bar{\sigma} \lambda_{0}, \quad q_{k} \sigma=\lambda_{0} p_{k} \bar{\sigma} \lambda_{0} . \\
\tilde{\lambda}_{0}^{z} & =\epsilon \tilde{\lambda}_{0}-z \tilde{y}, \\
\chi_{j}^{z} & =\chi_{j}+\frac{(\epsilon-1)\left\langle 0 p_{k} 0\right]-z\left\langle 0 p_{k} y\right]}{\left\langle 0 p_{j} p_{k} 0\right\rangle}\langle\mathbf{j} 0\rangle \lambda_{0}, \\
\chi_{k}^{z} & =\chi_{k}-\frac{(\epsilon-1)\left\langle 0 p_{j} 0\right]-z\left\langle 0 p_{j} y\right]}{\left\langle 0 p_{j} p_{k} 0\right\rangle}\langle\mathbf{k} 0\rangle \lambda_{0} .
\end{aligned}
$$

\section{B UV terms in Compton scattering}

We have seen in section 5 that, when spin $S$ of the matter particle is larger than one, the IR part of the Compton amplitude obtained via soft theorems develops an unphysical pole at $\left\langle 3 p_{1} 4\right] \rightarrow 0$. The same happens for $S>2$ in gravitational Compton scattering. The unphysical pole has to be canceled by the boundary term in the recursion relation, which we refer to as the $U V$ term in the discussion below. In this appendix we present an algorithm to construct a correct UV term, alternative to the one in ref. [34].

For this discussion we find it convenient to introduce the auxiliary objects:

$$
\begin{array}{rlrl}
\mathcal{A} & \equiv\langle\mathbf{1 3}\rangle[\mathbf{2} 4], \quad \mathcal{B} \equiv\langle\mathbf{2} 3\rangle[\mathbf{1 4}], & & \mathcal{X} \equiv m\langle\mathbf{1 2}\rangle+m[\mathbf{1 2}]-\left\langle\mathbf{1} p_{4} \mathbf{2}\right]-\left\langle\mathbf{2} p_{3} \mathbf{1}\right] \\
\mathcal{Y} \equiv\left(u-m^{2}\right) \mathcal{A}+\left(t-m^{2}\right) \mathcal{B}, & & \mathcal{Z} \equiv\left(u-m^{2}\right) \mathcal{A}-\left(t-m^{2}\right) \mathcal{B}
\end{array}
$$

They satisfy the important identities

$$
\left\langle 3 p_{1} 4\right] \mathcal{X}-\mathcal{Y}=2 m^{2}(\mathcal{A}+\mathcal{B}), \quad \mathcal{Z}=-\left\langle 3 p_{1} 4\right]\left(\left\langle\mathbf{1} p_{4} \mathbf{2}\right]+\left\langle\mathbf{2} p_{4} \mathbf{1}\right]\right) .
$$

\section{B.1 Electromagnetic Compton scattering}

The opposite helicity Compton scattering amplitude for matter particle $X$ of mass $m$ and spin $S$ can be written as $\mathcal{M}\left(\mathbf{1}_{X} \mathbf{2}_{\bar{X}} 3_{\gamma}^{-} 4_{\gamma}^{+}\right)=\mathcal{M}_{\mathrm{IR}}+\mathcal{M}_{\mathrm{UV}}$. The IR term takes the form [22]:

$$
\mathcal{M}_{\mathrm{IR}}=(-1)^{2 S} \frac{2 e^{2}\left\langle 3 p_{1} 4\right]^{2}}{\left(t-m^{2}\right)\left(u-m^{2}\right)}\left[\frac{\mathcal{A}+\mathcal{B}}{\left\langle 3 p_{1} 4\right]}\right]^{2 S}
$$

where $t=\left(p_{1}+p_{3}\right)^{2}, u=\left(p_{1}+p_{4}\right)^{2}$. As discussed in [22], the IR term fully accounts for the kinematic $t$ - and $u$-channel poles required by unitarity. The UV term can be zero or a pure contact term for $S \leq 1$. However, for $S>1$ the IR term develops an unphysical pole at $\left\langle 3 p_{1} 4\right] \rightarrow 0$. Therefore the UV term must be necessarily non-zero to cancel the unphysical pole, without screwing up the physical $t$ - and $u$-poles.

To understand the general formula for arbitrary spin $S$, it is useful start with a simpler example of $S=2$. The first identity in eq. (B.2) allows one to reshuffle the IR term, so as to rewrite the residue of the unphysical pole in a more convenient form [34]. Plugging it into eq. (B.3), the IR term for $S=2$ can be rewritten as

$$
\begin{aligned}
\mathcal{M}_{\mathrm{IR}} & =\frac{2 e^{2}\left\langle 3 p_{1} 4\right]^{2}}{\left(2 m^{2}\right)^{4}\left(t-m^{2}\right)\left(u-m^{2}\right)}\left[\frac{\left\langle 3 p_{1} 4\right] \mathcal{X}-\mathcal{Y}}{\left\langle 3 p_{1} 4\right]}\right]^{4} \\
& =\frac{2 e^{2}}{\left(2 m^{2}\right)^{4}\left(t-m^{2}\right)\left(u-m^{2}\right)}\left[\frac{\mathcal{Y}^{4}}{\left\langle 3 p_{1} 4\right]^{2}}-\frac{4 \mathcal{X} \mathcal{Y}^{3}}{\left\langle 3 p_{1} 4\right]}+\ldots\right],
\end{aligned}
$$


where the dots stand for terms with no unphysical poles. One can cancel the double pole in $\left\langle 3 p_{1} 4\right]$ by including in the $\mathcal{M}_{\mathrm{UV}}$ the term $-\frac{2 e^{2}}{\left(2 m^{2}\right)^{4}\left(t-m^{2}\right)\left(u-m^{2}\right)} \frac{\mathcal{Y}^{4}}{\left\langle 3 p_{1} 4\right]^{2}}$. This would however tamper with the $t$ - and $u$-poles, which we want to avoid. The trick is to compensate powers of $\mathcal{Y}$ with powers of $\mathcal{Z}$ which, by the second identity in eq. (B.2), do not affect the residue at $\left\langle 3 p_{1} 4\right] \rightarrow 0$. The crucial observation is that

$$
\frac{\mathcal{Y}^{2}-\mathcal{Z}^{2}}{\left(t-m^{2}\right)\left(u-m^{2}\right)}=4 \mathcal{A B}
$$

does not have a pole at $t \rightarrow m^{2}$ or $u \rightarrow m^{2}$. Therefore, a UV term proportional to $\frac{\mathcal{Y}^{2}-\mathcal{Z}^{2}}{\left(t-m^{2}\right)\left(u-m^{2}\right)}$ will affect the $\left\langle 3 p_{1} 4\right]$ poles without destroying the correct structure of physical poles provided by the IR term. In our case, the double pole at $\left\langle 3 p_{1} 4\right] \rightarrow 0$ is canceled if the UV term contains $\mathcal{M}_{\mathrm{UV}} \supset-\frac{2 e^{2}}{\left(2 m^{2}\right)^{4}\left(t-m^{2}\right)\left(u-m^{2}\right)} \frac{\mathcal{Y}^{4}-\mathcal{Z}^{4}}{\left\langle 3 p_{1} 4\right]^{2}}$. In exactly the same way we can deal with the single $\left\langle 3 p_{1} 4\right]$ pole in eq. (B.4). All in all, a legal UV term canceling all $\left\langle 3 p_{1} 4\right.$ ] poles in the $S=2$ Compton amplitude is given by

$$
\begin{aligned}
\mathcal{M}_{\mathrm{UV}} & =-\frac{2 e^{2}}{\left(2 m^{2}\right)^{4}\left(t-m^{2}\right)\left(u-m^{2}\right)}\left\{\frac{\mathcal{Y}^{2}\left(\mathcal{Y}^{2}-\mathcal{Z}^{2}\right)}{\left\langle 3 p_{1} 4\right]^{2}}-\frac{4 \mathcal{X} \mathcal{Y}\left(\mathcal{Y}^{2}-\mathcal{Z}^{2}\right)}{\left\langle 3 p_{1} 4\right]}\right\}+C \\
& =-\frac{e^{2} \mathcal{A B}}{2 m^{8}}\left\{\frac{\mathcal{Y}^{2}}{\left\langle 3 p_{1} 4\right]^{2}}-\frac{4 \mathcal{X} \mathcal{Y}}{\left\langle 3 p_{1} 4\right]}\right\}+C
\end{aligned}
$$

where $C$ is any pure contact term. This construction guarantees that $\mathcal{M}_{\mathrm{IR}}+\mathcal{M}_{\mathrm{UV}}$ has no unphysical poles, and that it has the same physical poles as $\mathcal{M}_{\mathrm{IR}}$. The UV term is ultraviolet in two ways. One, it scales as $\mathcal{O}\left(\epsilon^{1}\right)$ in the (complex kinematics) limit where $t \rightarrow m^{2}, u \rightarrow m^{2}$, and $s \rightarrow 0$ which is the limit where the photons become soft. This is unlike the IR term which scales as $\mathcal{O}\left(\epsilon^{-2}\right)$ in the soft limit. Two, $\mathcal{M}_{\mathrm{UV}}$ grows for $E \gg m$ as $\mathcal{O}\left(E^{8} / m^{8}\right)$, unlike the IR term, which is regular at high energies. This growth leads to a loss of perturbative unitarity at the scale $\Lambda$ close to the mass $m$ of the particle $X$, which is what ultimately prevents the possibility of fundamental spin-2 massive particles. In fact, we can further massage eq. (B.6) so as to improve the UV behavior by one notch, so as to (slightly) delay the onset of strong coupling. Using the first identity in eq. (B.2) to eliminate $\mathcal{Y}$, and absorbing the resulting $\mathcal{X}^{2}$ terms into $C$, we end up with

$$
\mathcal{M}_{\mathrm{UV}}=-\frac{2 e^{2} \mathcal{A} \mathcal{B}}{m^{6}}\left\{\frac{m^{2}(\mathcal{A}+\mathcal{B})^{2}}{\left\langle 3 p_{1} 4\right]^{2}}+\frac{\mathcal{X}(\mathcal{A}+\mathcal{B})}{\left\langle 3 p_{1} 4\right]}\right\}+C^{\prime}
$$

This has a better UV behavior, $\mathcal{M}_{\mathrm{UV}} \sim \mathcal{O}\left(E^{6} / m^{6}\right)$. Since the UV terms in eq. (B.6) and eq. (B.7) differ only by a contact term, they have exactly the same pole structure, and they both cancel the unphysical pole in $\mathcal{M}_{\mathrm{IR}}$.

Following exactly the same steps one can construct $\mathcal{M}_{\mathrm{UV}}$ for arbitrary $S>1$ :

$$
\mathcal{M}_{\mathrm{UV}}=-\frac{2 e^{2}}{\left(2 m^{2}\right)^{2 S}\left(t-m^{2}\right)\left(u-m^{2}\right)} \sum_{k=3}^{2 S}\left(\begin{array}{c}
2 S \\
k
\end{array}\right) \frac{(-\mathcal{X})^{2 S-k} \mathcal{Y}\left[\mathcal{Y}^{k-1}-\{\mathcal{Y} \| \mathcal{Z}\} \mathcal{Z}^{k-2}\right]}{\left\langle 3 p_{1} 4\right]^{k-2}}
$$

where $\{\mathcal{Y} \| \mathcal{Z}\}=\mathcal{Y}(\mathcal{Z})$ for even (odd) $k$. Note that $\mathcal{X}$ and $\mathcal{Z}$ are odd under $1 \leftrightarrow 2$, while $\mathcal{Y}$ is even. Therefore $\mathcal{M}_{\mathrm{UV}}$ has the same symmetry under $1 \leftrightarrow 2$ as the IR term: it is 
even (odd) for integer (half-integer) spin. The absence of physical poles in $\mathcal{M}_{\text {UV }}$ follows from $\mathcal{Y}^{n}-\mathcal{Z}^{n}=\left(\mathcal{Y}^{2}-\mathcal{Z}^{2}\right)\left(\mathcal{Y}^{n-2}+\mathcal{Y}^{n-4} \mathcal{Z}^{2}+\cdots+\mathcal{Z}^{n-2}\right)$ for any even $n \geq 2$. This is the most compact form of the UV term, but not the one with the best possible UV behavior. As in the case of $S=2$, we can always soften the UV behavior from $\mathcal{O}\left(E^{4 S} / m^{4 S}\right)$ down to $\mathcal{O}\left(E^{4 S-2} / m^{4 S-2}\right)$ by using the first identity in eq. (B.2) and isolating the resulting $\mathcal{O}\left(E^{4 S} / m^{4 S}\right)$ contact term. The consequence is that the maximum cutoff scale of an effective theory describing a spin- $S>1$ particle with mass $m$ and electric charge $q$ satisfies

$$
\Lambda_{\max } \lesssim\left(\frac{4 \pi}{|q| e}\right)^{\frac{1}{2 S-1}}
$$

This bound was obtained by other methods in ref. [53].

For Compton scattering via the electromagnetic dipole the IR amplitude can be read off from eq. (5.9):

$$
\mathcal{M}_{\mathrm{IR}}=|a|^{2} \frac{(-1)^{2 S} S e^{2}}{2 m^{2}\left(t-m^{2}\right)\left(u-m^{2}\right)}\left[\frac{\mathcal{A}+\mathcal{B}}{\left\langle 3 p_{1} 4\right]}\right]^{2 S-2}\{(\mathcal{Y}+\mathcal{Z})(\mathcal{A}+2 S \mathcal{B})+(\mathcal{Y}-\mathcal{Z})(2 S \mathcal{A}+\mathcal{B})\} .
$$

The corresponding UV term can be easily constructed by the same techniques:

$$
\begin{aligned}
\mathcal{M}_{\mathrm{UV}}= & -|a|^{2} \frac{S e^{2}}{\left(2 m^{2}\right)^{2 S-1}\left(t-m^{2}\right)\left(u-m^{2}\right)} \sum_{k=1}^{2 S-2}\left(\begin{array}{c}
2 S-2 \\
k
\end{array}\right) \frac{(-X)^{2 S-2-k}}{\left\langle 3 p_{1} 4\right]^{k}} \\
& \times\left\{\left(\mathcal{Y}^{k}-\mathcal{Z}^{k}\right)(\mathcal{Y}+\mathcal{Z})(\mathcal{A}+2 S \mathcal{B})+\left(\mathcal{Y}^{k}-(-\mathcal{Z})^{k}\right)(\mathcal{Y}-\mathcal{Z})(2 S \mathcal{A}+\mathcal{B})\right\} .
\end{aligned}
$$

\section{B.2 Gravitational Compton scattering}

We turn to the analogue of the Compton process in gravity, where photons are replaced by massless spin-2 gravitons. We assume minimal gravitational interactions of a spin$\mathrm{S}$ massive particle $X$. We focus on the opposite helicity Compton amplitude and again separate the UV and IR terms: $\mathcal{M}\left(\mathbf{1}_{X} \mathbf{2}_{X} 3_{h}^{-} 4_{h}^{+}\right)=\mathcal{M}_{\mathrm{IR}}+\mathcal{M}_{\mathrm{UV}}$. The IR term takes the form [22]:

$$
\begin{aligned}
\mathcal{M}_{\mathrm{IR}} & =\frac{(\mathcal{A}+\mathcal{B})^{4}}{M_{\mathrm{Pl}}^{2} s\left(t-m^{2}\right)\left(u-m^{2}\right)}\left[\frac{\mathcal{A}+\mathcal{B}}{\left\langle 3 p_{1} 4\right]}\right]^{2 S-4} \\
& =\frac{\mathcal{A}+\mathcal{B}}{M_{\mathrm{Pl}}^{2}\left(2 m^{2}\right)^{2 S-2}} \sum_{k=0}^{2 S-3}(-1)^{k}\left(\begin{array}{c}
2 S-3 \\
k
\end{array}\right) X^{2 S-3-k} I_{k}
\end{aligned}
$$

where we abbreviated

$$
I_{k} \equiv \frac{2 m^{2}(\mathcal{A}+\mathcal{B})^{2}}{s\left(t-m^{2}\right)\left(u-m^{2}\right)} \frac{\mathcal{Y}^{k}}{\left\langle 3 p_{1} 4\right]^{k-1}},
$$

and $\mathcal{A}, \mathcal{B}, \mathcal{X}, \mathcal{Y}$ are defined in eq. (B.1). For $S>2$ the IR term in eq. (B.12) develops a pole at $\left\langle 3 p_{1} 4\right] \rightarrow 0$, therefore $\mathcal{M}_{\mathrm{UV}}$ has to be non-zero for $S>2$ so as to cancel the unphysical pole. Below we construct a befitting UV term. We will first find a set of functions $U_{k}$ such 
that $U_{k}+I_{k}$ is regular at $\left\langle 3 p_{1} 4\right] \rightarrow 0$ and has the same kinematic poles as $I_{k}$. Given such a set, a legal UV term for $S>2$ is simply

$$
\mathcal{M}_{\mathrm{UV}}=\frac{\mathcal{A}+\mathcal{B}}{M_{\mathrm{Pl}}^{2}\left(2 m^{2}\right)^{2 S-2}} \sum_{k=2}^{2 S-3}(-1)^{k}\left(\begin{array}{c}
2 S-3 \\
k
\end{array}\right) X^{2 S-3-k} U_{k} .
$$

We will construct $U_{k}$ recursively. In this endeavour the following identity is instrumental:

$$
(\mathcal{A}+\mathcal{B})^{2}=\frac{1}{m^{2}}\left\{s \mathcal{A B}+m\left\langle 3 p_{1} 4\right](\mathcal{A}+\mathcal{B})(\langle\mathbf{1 2}\rangle+[\mathbf{1 2}])-\left\langle 3 p_{1} 4\right]^{2}\langle\mathbf{1 2}\rangle[\mathbf{1 2}]\right\} .
$$

This allows us to rewrite $I_{k}$ as

$$
I_{k}=\frac{2 \mathcal{A B}}{\left(t-m^{2}\right)\left(u-m^{2}\right)} \frac{\mathcal{Y}^{k}}{\left\langle 3 p_{1} 4\right]^{k-1}}+c_{k}(\langle\mathbf{1 2}\rangle+[\mathbf{1 2}])-d_{k}\langle\mathbf{1 2}\rangle[\mathbf{1 2}],
$$

where once again we introduced some auxiliary notation:

$$
c_{k} \equiv \frac{2 m(\mathcal{A}+\mathcal{B})}{s\left(t-m^{2}\right)\left(u-m^{2}\right)} \frac{\mathcal{Y}^{k}}{\left\langle 3 p_{1} 4\right]^{k-2}}, \quad d_{k} \equiv \frac{2}{s\left(t-m^{2}\right)\left(u-m^{2}\right)} \frac{\mathcal{Y}^{k}}{\left\langle 3 p_{1} 4\right]^{k-3}} .
$$

We can rearrange $c_{k}$ as

$$
c_{k}=\frac{2 m(\mathcal{A}+\mathcal{B})}{s\left(t-m^{2}\right)\left(u-m^{2}\right)} \frac{\mathcal{Y}^{k-1}}{\left\langle 3 p_{1} 4\right]^{k-2}}\left[-2 m^{2}(\mathcal{A}+\mathcal{B})+\mathcal{X}\left\langle 3 p_{1} 4\right]\right]=-2 m I_{k-1}+\mathcal{X} c_{k-1} .
$$

This recursive equation is solved by

$$
c_{k}=-2 m \sum_{l=1}^{k-2} I_{k-l} \mathcal{X}^{l-1}+\mathcal{X}^{k-2} c_{2} .
$$

We can handle $d_{k}$ in a similar way

$$
d_{k} \equiv \frac{2}{s\left(t-m^{2}\right)\left(u-m^{2}\right)} \frac{\mathcal{Y}^{k-2}}{\left\langle 3 p_{1} 4\right]^{k-3}}\left[-2 m^{2}(\mathcal{A}+\mathcal{B})+\mathcal{X}\left\langle 3 p_{1} 4\right]\right]^{2}=4 m^{2} I_{k-2}-4 m^{2} \mathcal{X}_{c_{k-2}}+\mathcal{X}^{2} d_{k-2}
$$

The solution is

$$
\begin{aligned}
& d_{k}=4 m^{2} \sum_{l=1}^{(k-2) / 2}\left(I_{k-2 l}-\mathcal{X}_{c_{k-2 l}}\right) \mathcal{X}^{2(l-1)}+\mathcal{X}^{k-2} d_{2}, \quad \text { even } k, \\
& d_{k}=4 m^{2} \sum_{l=1}^{(k-3) / 2}\left(I_{k-2 l}-\mathcal{X}_{c_{k-2 l}}\right) \mathcal{X}^{2(l-1)}+\mathcal{X}^{k-3} d_{3}, \quad \text { odd } k .
\end{aligned}
$$

Plugging the solution back into the formula for $I_{k}$ we obtain

$$
\begin{aligned}
I_{k}= & \frac{2 \mathcal{A B}}{\left(t-m^{2}\right)\left(u-m^{2}\right)} \frac{\mathcal{Y}^{k}}{\left\langle 3 p_{1} 4\right]^{k-1}}-2 m(\langle\mathbf{1 2}\rangle+[\mathbf{1 2}]) \sum_{l=1}^{k-2} I_{k-l} \mathcal{X}^{l-1} \\
& -4 m^{2}\langle\mathbf{1 2}\rangle[\mathbf{1 2}] \sum_{l=1}^{(k-\{2|| 3\}) / 2}\left(I_{k-2 l}+2 m \mathcal{X} \sum_{n=1}^{k-2 l-2} I_{k-n} \mathcal{X}^{n-1}\right) \mathcal{X}^{2(l-1)}+\ldots
\end{aligned}
$$


where the dots stand for $d_{2,3}$ and $c_{2}$ terms, which do not have poles at $\left\langle 3 p_{1} 4\right]$. We are ready to write down a recursive equation for $U_{k}$ :

$$
\begin{aligned}
U_{k}= & -\frac{2 \mathcal{A B}}{\left(t-m^{2}\right)\left(u-m^{2}\right)} \frac{\mathcal{Y}^{k}-\{\mathcal{Z} \| Y\} \mathcal{Z}^{k-1}}{\left\langle 3 p_{1} 4\right]^{k-1}}-2 m(\langle\mathbf{1 2}\rangle+[\mathbf{1 2}]) \sum_{l=1}^{k-2} U_{k-l} \mathcal{X}^{l-1} \\
& -4 m^{2}\langle\mathbf{1 2}\rangle[\mathbf{1 2}] \sum_{l=1}^{(k-\{2 \| 3\}) / 2}\left(U_{k-2 l}+2 m \mathcal{X} \sum_{n=1}^{k-2 l-2} U_{k-n} \mathcal{X}^{n-1}\right) \mathcal{X}^{2(l-1)}
\end{aligned}
$$

where $\{\mathcal{Z} \| Y\}=\mathcal{Z}(\mathcal{Y})$ for even (odd) $k$, and $\{2 \| 3\}=2(3)$ for even (odd) $l$. An implicit convention above is that, if the upper limit of a summation is smaller than the lower limit, then the whole sum is void.

The recursive equation (B.23) supplemented by the boundary condition $U_{0}=U_{1}=0$ defines $U_{k}$ for arbitrary $k$. Via eq. (B.14), this allows one to construct an appropriate UV term for any spin $S$ of the matter particle. Let us see how it works in some simple examples. For $k=2$ we get

$$
U_{2}=-\frac{2 \mathcal{A B}}{\left(t-m^{2}\right)\left(u-m^{2}\right)} \frac{\mathcal{Y}^{2}-\mathcal{Z}^{2}}{\left\langle 3 p_{1} 4\right]}=-\frac{8 \mathcal{A}^{2} \mathcal{B}^{2}}{\left\langle 3 p_{1} 4\right]}
$$

By eq. (B.14), the UV term for $S=5 / 2$ reads

$$
\mathbf{S}=\mathbf{5} / \mathbf{2}: \quad \mathcal{M}_{\mathrm{UV}}=\frac{\mathcal{A}+\mathcal{B}}{M_{\mathrm{Pl}}^{2}\left(2 m^{2}\right)^{3}} U_{2}=-\frac{\mathcal{A}^{2} \mathcal{B}^{2}(\mathcal{A}+\mathcal{B})}{M_{\mathrm{Pl}}^{2} m^{6}\left\langle 3 p_{1} 4\right]} .
$$

For $k=3$ eq. (B.23) yields

$$
U_{3}=-8 \mathcal{A}^{2} \mathcal{B}^{2}\left(\frac{\mathcal{Y}}{\left\langle 3 p_{1} 4\right]^{2}}-\frac{2 m(\langle\mathbf{1 2}\rangle+[\mathbf{1 2}])}{\left\langle 3 p_{1} 4\right]}\right) .
$$

By eq. (B.14), the UV term for $S=3$ reads

$$
\begin{aligned}
\mathcal{M}_{\mathrm{UV}} & =\frac{\mathcal{A}+\mathcal{B}}{M_{\mathrm{Pl}}^{2}\left(2 m^{2}\right)^{4}}\left(3 \mathcal{X} U_{2}-U_{3}\right) \\
& =-\frac{\mathcal{A}^{2} \mathcal{B}^{2}(\mathcal{A}+\mathcal{B})}{M_{\mathrm{Pl}}^{2} m^{8}}\left(\frac{\mathcal{X}}{\left\langle 3 p_{1} 4\right]}+\frac{m(\langle\mathbf{1 2}\rangle+[\mathbf{1 2}])}{\left\langle 3 p_{1} 4\right]}+\frac{m^{2}(\mathcal{A}+\mathcal{B})}{\left\langle 3 p_{1} 4\right]^{2}}\right) .
\end{aligned}
$$

And so on.

From eq. (B.23) one can read off that $U_{k}$ scales as $\mathcal{O}\left(E^{2(k+1)}\right)$ at large energies. For Compton processes with energies much above the particle's mass one thus finds that the UV term grows with energy as

$$
\mathcal{M}_{\mathrm{UV}} \sim \mathcal{O}\left(\frac{E^{4 S-2}}{m^{4 S-4} M_{\mathrm{Pl}}^{2}}\right), \quad E \gg m,
$$

for any $S>2$. This violates perturbative unitarity at some energy scale above the particle's mass. One concludes that the maximum cutoff scale for an effective theory describing a particle of mass $m$ and spin $S>2$ satisfies

$$
\Lambda_{\max } \lesssim\left(4 \pi M_{\mathrm{Pl}} m^{2 S-2}\right)^{\frac{1}{2 S-1}} .
$$


This bound was conjectured in refs. [28, 54]. The bound is totally general and universal, since every particle has to be coupled to gravity. As the spin increases, the maximum cutoff quickly approaches $m$, squeezing the validity range of the effective theory to a very narrow energy interval. This imposes severe restrictions on constructing viable effective theories of higher-spin particles (see also refs. $[55,56]$ for other arguments leading to similar conclusions).

Open Access. This article is distributed under the terms of the Creative Commons Attribution License (CC-BY 4.0), which permits any use, distribution and reproduction in any medium, provided the original author(s) and source are credited.

\section{References}

[1] F.E. Low, Bremsstrahlung of very low-energy quanta in elementary particle collisions, Phys. Rev. 110 (1958) 974 [INSPIRE].

[2] S. Weinberg, Infrared photons and gravitons, Phys. Rev. 140 (1965) B516 [InSPIRE].

[3] J. Broedel, M. de Leeuw, J. Plefka and M. Rosso, Constraining subleading soft gluon and graviton theorems, Phys. Rev. D 90 (2014) 065024 [arXiv:1406.6574] [INSPIRE].

[4] L. Rodina, Scattering amplitudes from soft theorems and infrared behavior, Phys. Rev. Lett. 122 (2019) 071601 [arXiv:1807.09738] [INSPIRE].

[5] A. Strominger, Lectures on the infrared structure of gravity and gauge theory, arXiv: 1703.05448 [INSPIRE].

[6] C. Cheung, K. Kampf, J. Novotny, C.-H. Shen and J. Trnka, On-shell recursion relations for effective field theories, Phys. Rev. Lett. 116 (2016) 041601 [arXiv: 1509.03309] [INSPIRE].

[7] C. Cheung, K. Kampf, J. Novotny, C.-H. Shen and J. Trnka, A periodic table of effective field theories, JHEP 02 (2017) 020 [arXiv: 1611.03137] [InSPIRE].

[8] C. Cheung, K. Kampf, J. Novotny, C.-H. Shen, J. Trnka and C. Wen, Vector effective field theories from soft limits, Phys. Rev. Lett. 120 (2018) 261602 [arXiv:1801.01496] [INSPIRE].

[9] H. Elvang, M. Hadjiantonis, C.R.T. Jones and S. Paranjape, Soft bootstrap and supersymmetry, JHEP 01 (2019) 195 [arXiv: 1806. 06079] [INSPIRE].

[10] I. Low and Z. Yin, Soft bootstrap and effective field theories, JHEP 11 (2019) 078 [arXiv: 1904.12859] [INSPIRE].

[11] F. Cachazo and A. Strominger, Evidence for a new soft graviton theorem, arXiv:1404.4091 [INSPIRE].

[12] R. Britto, F. Cachazo, B. Feng and E. Witten, Direct proof of tree-level recursion relation in Yang-Mills theory, Phys. Rev. Lett. 94 (2005) 181602 [hep-th/0501052] [INSPIRE].

[13] Z. Bern, L.J. Dixon, M. Perelstein and J.S. Rozowsky, Multileg one loop gravity amplitudes from gauge theory, Nucl. Phys. B 546 (1999) 423 [hep-th/9811140] [INSPIRE].

[14] S. He, Y.-T. Huang and C. Wen, Loop corrections to soft theorems in gauge theories and gravity, JHEP 12 (2014) 115 [arXiv:1405.1410] [INSPIRE].

[15] B. Sahoo and A. Sen, Classical and quantum results on logarithmic terms in the soft theorem in four dimensions, JHEP 02 (2019) 086 [arXiv: 1808.03288] [INSPIRE].

[16] H. Elvang, C.R.T. Jones and S.G. Naculich, Soft photon and graviton theorems in effective field theory, Phys. Rev. Lett. 118 (2017) 231601 [arXiv:1611.07534] [INSPIRE]. 
[17] M. Bianchi and A.L. Guerrieri, On the soft limit of closed string amplitudes with massive states, Nucl. Phys. B 905 (2016) 188 [arXiv:1512.00803] [INSPIRE].

[18] S. Atul Bhatkar and B. Sahoo, Subleading soft theorem for arbitrary number of external soft photons and gravitons, JHEP 01 (2019) 153 [arXiv:1809.01675] [INSPIRE].

[19] A. Sen, Subleading soft graviton theorem for loop amplitudes, JHEP 11 (2017) 123 [arXiv: 1703.00024] [INSPIRE].

[20] A. Laddha and A. Sen, Sub-subleading soft graviton theorem in generic theories of quantum gravity, JHEP 10 (2017) 065 [arXiv:1706.00759] [INSPIRE].

[21] S. Chakrabarti, S.P. Kashyap, B. Sahoo, A. Sen and M. Verma, Subleading soft theorem for multiple soft gravitons, JHEP 12 (2017) 150 [arXiv:1707.06803] [INSPIRE].

[22] N. Arkani-Hamed, T.-C. Huang and Y.-T. Huang, Scattering amplitudes for all masses and spins, arXiv:1709.04891 [INSPIRE].

[23] D.A. Kosower, B. Maybee and D. O'Connell, Amplitudes, observables, and classical scattering, JHEP 02 (2019) 137 [arXiv:1811.10950] [INSPIRE].

[24] A. Guevara, A. Ochirov and J. Vines, Scattering of spinning black holes from exponentiated soft factors, JHEP 09 (2019) 056 [arXiv: 1812.06895] [INSPIRE].

[25] A. Laddha and A. Sen, Gravity waves from soft theorem in general dimensions, JHEP 09 (2018) 105 [arXiv: 1801.07719] [INSPIRE].

[26] N. Arkani-Hamed, Y.-T. Huang and D. O'Connell, Kerr black holes as elementary particles, JHEP 01 (2020) 046 [arXiv:1906.10100] [INSPIRE].

[27] J. Bonifacio and K. Hinterbichler, Bounds on amplitudes in effective theories with massive spinning particles, Phys. Rev. D 98 (2018) 045003 [arXiv:1804.08686] [INSPIRE].

[28] J. Bonifacio and K. Hinterbichler, Universal bound on the strong coupling scale of a gravitationally coupled massive spin-2 particle, Phys. Rev. D 98 (2018) 085006 [arXiv: 1806.10607] [INSPIRE].

[29] J. Bonifacio, K. Hinterbichler and R.A. Rosen, Constraints on a gravitational Higgs mechanism, Phys. Rev. D 100 (2019) 084017 [arXiv:1903.09643] [InSPIRE].

[30] A. Falkowski and G. Isabella, Matter coupling in massive gravity, JHEP 04 (2020) 014 [arXiv:2001.06800] [INSPIRE].

[31] Y. Shadmi and Y. Weiss, Effective field theory amplitudes the on-shell way: scalar and vector couplings to gluons, JHEP 02 (2019) 165 [arXiv:1809.09644] [INSPIRE].

[32] R. Aoude and C.S. Machado, The rise of SMEFT on-shell amplitudes, JHEP 12 (2019) 058 [arXiv: 1905.11433] [INSPIRE].

[33] G. Durieux, T. Kitahara, Y. Shadmi and Y. Weiss, The electroweak effective field theory from on-shell amplitudes, JHEP 01 (2020) 119 [arXiv:1909.10551] [INSPIRE].

[34] M.-Z. Chung, Y.-T. Huang, J.-W. Kim and S. Lee, The simplest massive S-matrix: from minimal coupling to black holes, JHEP 04 (2019) 156 [arXiv: 1812.08752] [INSPIRE].

[35] A. Guevara, A. Ochirov and J. Vines, Black-hole scattering with general spin directions from minimal-coupling amplitudes, Phys. Rev. D 100 (2019) 104024 [arXiv:1906.10071] [INSPIRE].

[36] C. Cheung, A. de la Fuente and R. Sundrum, $4 D$ scattering amplitudes and asymptotic symmetries from $2 D$ CFT, JHEP 01 (2017) 112 [arXiv:1609.00732] [INSPIRE]. 
[37] S. Pasterski and S.-H. Shao, Conformal basis for flat space amplitudes, Phys. Rev. D 96 (2017) 065022 [arXiv:1705.01027] [INSPIRE].

[38] L. Donnay, A. Puhm and A. Strominger, Conformally soft photons and gravitons, JHEP 01 (2019) 184 [arXiv: 1810.05219] [inSPIRE].

[39] H.K. Dreiner, H.E. Haber and S.P. Martin, Two-component spinor techniques and Feynman rules for quantum field theory and supersymmetry, Phys. Rept. 494 (2010) 1 [arXiv: 0812.1594] [INSPIRE].

[40] S. Dittmaier, Weyl-van der Waerden formalism for helicity amplitudes of massive particles, Phys. Rev. D 59 (1998) 016007 [hep-ph/9805445] [INSPIRE].

[41] E. Conde and A. Marzolla, Lorentz constraints on massive three-point amplitudes, JHEP 09 (2016) 041 [arXiv: 1601.08113] [INSPIRE].

[42] R. Franken and C. Schwinn, On-shell constructibility of Born amplitudes in spontaneously broken gauge theories, JHEP 02 (2020) 073 [arXiv: 1910.13407] [INSPIRE].

[43] S. Weinberg, Photons and gravitons in S-matrix theory: derivation of charge conservation and equality of gravitational and inertial mass, Phys. Rev. 135 (1964) B1049 [InSPIRE].

[44] E. Witten, Perturbative gauge theory as a string theory in twistor space, Commun. Math. Phys. 252 (2004) 189 [hep-th/0312171] [INSPIRE].

[45] R.O. Hansen, Multipole moments of stationary space-times, J. Math. Phys. 15 (1974) 46 [INSPIRE].

[46] A. Guevara, Notes on conformal soft theorems and recursion relations in gravity, arXiv: 1906.07810 [INSPIRE].

[47] W.-M. Chen, Y.-T. Huang and C. Wen, New fermionic soft theorems for supergravity amplitudes, Phys. Rev. Lett. 115 (2015) 021603 [arXiv:1412.1809] [INSPIRE].

[48] D. Jain and A. Rudra, Leading soft theorem for multiple gravitini, JHEP 06 (2019) 004 [arXiv: 1811.01804] [INSPIRE].

[49] T. Klose, T. McLoughlin, D. Nandan, J. Plefka and G. Travaglini, Double-soft limits of gluons and gravitons, arXiv:1504.05558 [INSPIRE].

[50] S. Chakrabarti, S.P. Kashyap, B. Sahoo, A. Sen and M. Verma, Testing subleading multiple soft graviton theorem for CHY prescription, JHEP 01 (2018) 090 [arXiv:1709.07883] [INSPIRE].

[51] Y.T.A. Law and M. Zlotnikov, Massive spinning bosons on the celestial sphere, JHEP 06 (2020) 079 [arXiv: 2004.04309] [INSPIRE].

[52] H. Johansson and A. Ochirov, Double copy for massive quantum particles with spin, JHEP 09 (2019) 040 [arXiv: 1906.12292] [INSPIRE].

[53] M. Porrati and R. Rahman, A model independent ultraviolet cutoff for theories with charged massive higher spin fields, Nucl. Phys. B $\mathbf{8 1 4}$ (2009) 370 [arXiv:0812.4254] [INSPIRE].

[54] R. Rahman, Interacting massive higher spin fields, Ph.D. thesis, New York University, New York, NY, U.S.A. (2009).

[55] S. Caron-Huot, Z. Komargodski, A. Sever and A. Zhiboedov, Strings from massive higher spins: the asymptotic uniqueness of the Veneziano amplitude, JHEP 10 (2017) 026 [arXiv: 1607.04253] [INSPIRE].

[56] N. Afkhami-Jeddi, S. Kundu and A. Tajdini, A bound on massive higher spin particles, JHEP 04 (2019) 056 [arXiv:1811.01952] [INSPIRE]. 